\title{
Gradhiva
}

\section{Une illusion du vraisemblable. Mise en scène taphonomique et prospective néolithique à Çatalhöyük}

An illusion of likelihood. Taphonomic staging and Neolithic prospective at Çatalhöyük

\section{Rémi Hadad}

\section{OpenEdition}

Journals

Édition électronique

URL : http://journals.openedition.org/gradhiva/3750

DOI : 10.4000/gradhiva.3750

ISSN : 1760-849X

\section{Éditeur}

Musée du quai Branly Jacques Chirac

\section{Édition imprimée}

Date de publication : 28 novembre 2018

Pagination : 112-141

ISBN : 978-2-35744-111-8

ISSN : 0764-8928

\section{Référence électronique}

Rémi Hadad, « Une illusion du vraisemblable. Mise en scène taphonomique et prospective néolithique à Çatalhöyük », Gradhiva [En ligne], 28 | 2018, mis en ligne le 28 novembre 2020, consulté le 06 janvier 2021. URL : http://journals.openedition.org/gradhiva/3750 ; DOI : https://doi.org/10.4000/gradhiva. 3750 

Une fllusion du vraisemblable

Mise en scène taphonomique et prospective néolithique à Çatalhöyük

par Rémi Hadad

Le site néolithique de Çatalhöyük, en Anatolie, tire sa notoriété des peintures murales et des espaces architecturaux magnifiquement préservés qui lui confèrent parfois un air de Pompéi préhistorique. Tout comme la célèbre ville antique, cet état de conservation résulte d'une destruction soudaine, soustrayant les vestiges aux processus irrévocables d'érosion ou de démolition. Çatalhöyük, toutefois, n'a pas été frappé par une catastrophe unique et définitive mais est le résultat de pratiques culturelles qui ont inhumé et accumulé ces restes tout au long de l'occupation du site. La notion de capsule temporelle conjure l'illusion qui consisterait à y voir, comme à Pompéi, des instantanés de la vie quotidienne, pour saisir au contraire la logique de ses condamnations volontaires et le statut interprétatif des vestiges ainsi constitués. Produire activement du passé, pour le dire vite, c'est aussi agir au futur.

mots clés

archéologie, temporalité,

architecture, futur, Néolithique 
1. Les rapports de mission sont tous disponibles sur le site du Çatalhöyük Research Project: www. catalhoyuk.com/research.
Cinq années seulement après son exhumation, et bien qu'il ait été protégé des intempéries par un nouvel abri, le bâtiment 52 de Çatalhöyük avait perdu une bonne partie de sa capacité d'évocation (fig. 1). Les angles des murs en adobe s'étaient émoussés et les sols enduits s'étaient effrités. Leurs courbes si caractéristiques étaient recouvertes d'une épaisse couche de poussière argileuse, résultat cumulé des dépôts amenés par les vents qui traversent sans obstacle la plaine de Konya et des résidus de l'inexorable délitement du bâti. Le dôme du four en tannour intégré à l'un des murs était effondré, et il n'y avait guère que le crâne d'aurochs inséré dans une alcôve pour continuer d'incarner - à peine discernable derrière une pile de sacs de sable la richesse des «maisons" néolithiques passées à la postérité avec les photographies et reconstitutions de l'inventeur du site, James Mellaart (1967).

Comment conserver ce qui n'a pas été construit pour durer, du moins pas sous cette forme, pas sans les réfections et les apports réguliers en matériaux que nécessite ce type d'architecture en terre? À Çatalhöyük, depuis la reprise des fouilles dans les années 1990 (Hodder 2006), et plus encore depuis l'inscription du site sur la liste du Patrimoine mondial de I'Unesco en 2012 et l'augmentation du nombre de visiteurs, une équipe de conservateurs s'affaire à concilier logiques archéologiques et patrimoniales afin d'en préserver l'intérêt visuel. Concernant le bâtiment 52, cependant, le balayage annuel n'y suffira pas: il faut "descendre», enlever les couches successives d'enduits et de remblais, ainsi que les murs et les installations ajoutées au cours de l'occupation, et ce dans l'ordre strictement inverse de leur construction, afin de mettre au jour une phase précédente de la biographie du bâtiment (ou un bâtiment sous-jacent). Non seulement la puissance évocatrice des vestiges s'en trouvera ravivée, mais la fouille permettra de tester les hypothèses émises sur cette "maison» lors de son exhumation et d'éclairer les conditions de sa destruction par un incendie, sans doute volontaire, au milieu du vII ${ }^{\mathrm{e}}$ millénaire avant notre ère (Twiss et al. 2008; Farid 2014).

Pendant trois saisons, de 2013 à 2015, je me trouvais ainsi chargé de coordonner la fouille du bâtiment 52 et de ses sépultures situées, comme il est d'usage à Çatalhöyük, sous les plateformes de la partie nord de la pièce centrale ${ }^{1}$. Leur emplacement était devenu particulièrement flagrant après que l'érosion eut creusé des dépressions indiquant, sous la surface, les fosses remplies d'un autre sédiment que le bâti lui-même. Les photographies des dernières fouilles montrèrent en outre que l'une de ces tombes n'avait jamais été recouverte d'enduit comme cela est le cas lorsque le bâtiment continue d'être habité. La fouille devait donc s'y focaliser en priorité puisqu'il s'agissait de l'une des dernières actions des occupants avant l'incendie. Rapidement, une terre riche en particules fibreuses apparut sous la truelle, d'un type qui laisse habituellement penser à une contamination récente par un terrier ou des racines. Mais la disposition des squelettes et la forme intacte de la fosse levèrent toute équivoque.

Protégée par son réceptacle argileux, la sépulture avait en fait "cuit»" dans l'incendie du bâtiment. Certains des matériaux organiques avaient ainsi été en partie conservés. Un bol en bois couvrait le crâne d'un jeune enfant recroquevillé sur l'épaule gauche d'un homme adulte inhumé, quant à lui, sur le dos, au fond de la fosse, les genoux repliés sur la poitrine (fig. 2). La dépouille d'un nouveau-né avait été déposée juste au-dessus de l'enfant, de manière à ce que les têtes des trois individus soient en contact. 


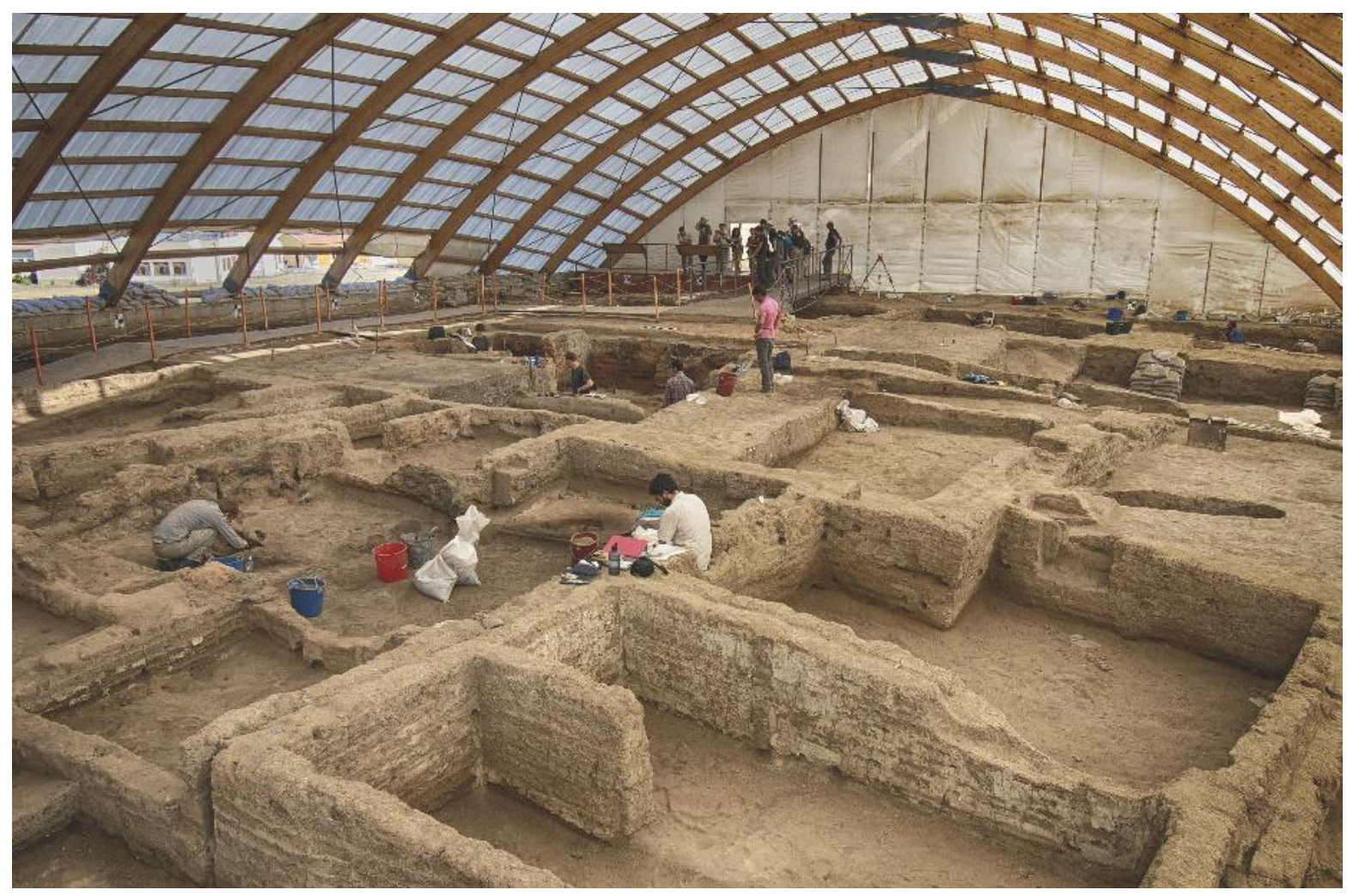

Les squelettes superposés des deux jeunes défunts étaient séparés par les restes d'une toile en lin qui servit sans doute de linceul au nourrisson placé en dernier dans la fosse. Les restes très incomplets de six autres enfants, dont les corps devaient se trouver à différents degrés de décomposition au moment de leur dépôt, accompagnaient l'ensemble (Haddow et al. 2016). Des résidus plus ou moins terreux d'origine organique se concentraient à l'intérieur de la cavité crânienne et au niveau de l'abdomen des individus les plus exposés à la chaleur de l'incendie.

Ce genre de découverte frappe d'un sentiment de réalité auquel la fouille sur des sites à la matérialité sèche, stérile, ne prépare pas complètement. De tels contextes avaient déjà fourni à Mellaart certains des artefacts les plus spectaculaires illustrant le panorama de la vie quotidienne qu'il dépeint dans sa monographie grand public (1967: 208-211). Mais un tel degré de «résolution» est resté rare depuis la reprise des fouilles. La sépulture multiple est du reste une pratique funéraire inhabituelle à Çatalhöyük (Boz et Hager 2013). Tout cela questionnait le lien entre ce dépôt si particulier et les circonstances de l'incendie du bâtiment. Cette expérience inaugurale me conduisit donc à reconsidérer les relations complexes qui unissent - de manière plus générale destruction et conservation à Çatalhöyük. Malgré son caractère singulier, la scène funéraire du bâtiment 52 a en effet ceci de paradigmatique qu'elle illustre toute l'ambivalence de la préservation à la fois exceptionnelle et transitoire du site: la difficulté contemporaine à en conserver les parties exposées est la conséquence de vestiges qui sont moins le résultat d'une résistance fig. 1

Reprise des fouilles sur le secteur nord du site archéologique de Çatalhöyük, Turquie, 2013. Après plusieurs années d'exposition au public, les bâtiments exhumés montrent des signes avancés de délitement. On distingue, au premier plan, l'émiettement de l'enduit blanc et l'érosion en affouillement à la base des murs. Au deuxième plan, le bâtiment 52 et ses sépultures en cours de fouille.

Photo @ Jason Quinlan. 


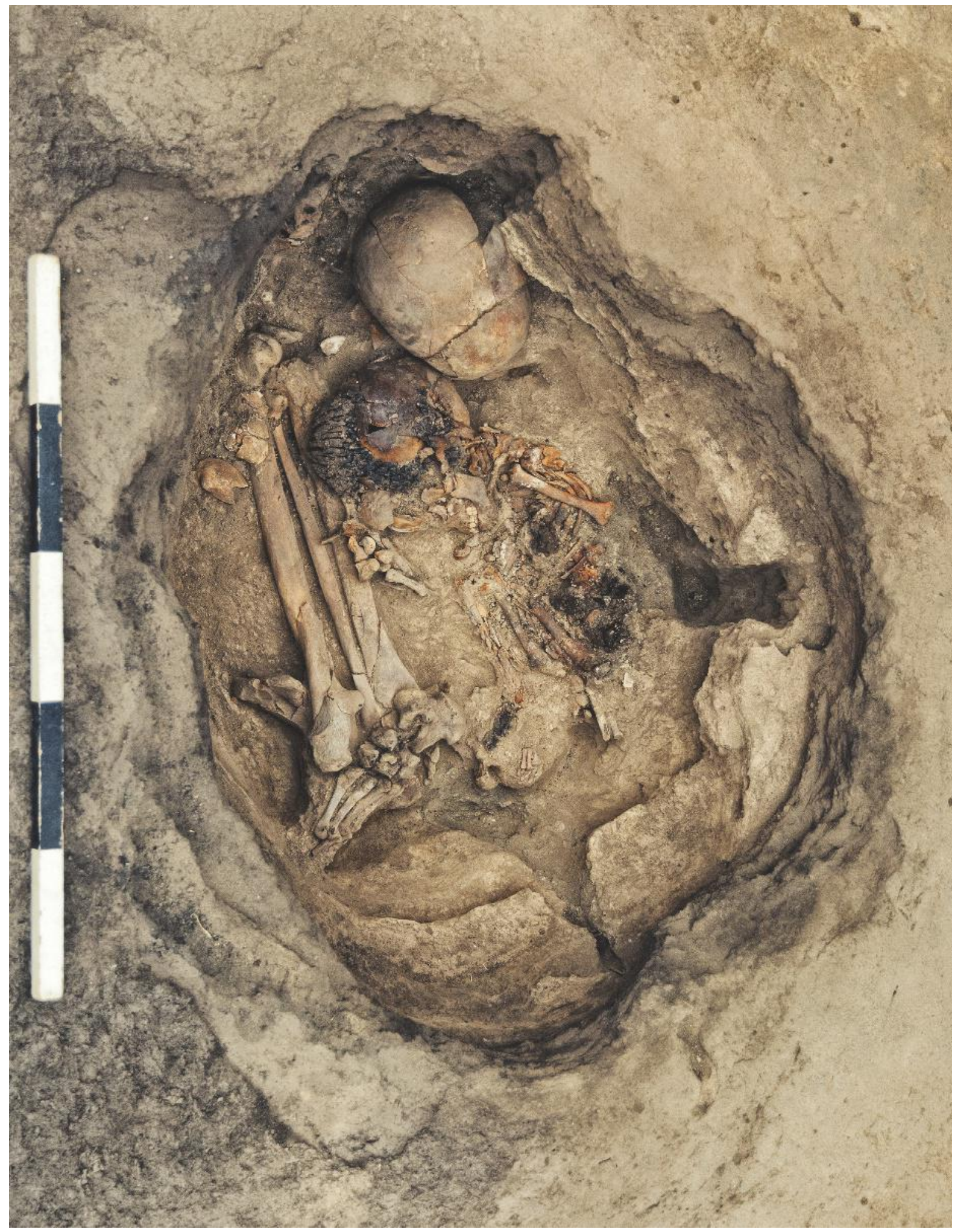


aux épreuves du temps que d'épisodes d'enfouissement rapides et définitifs. À la fin de la période d'occupation de chaque bâtiment, la partie supérieure de la structure est démolie, ce qu'il en reste est comblé par les débris et du remblai, avant qu'un nouveau bâtiment ne vienne généralement coiffer l'ensemble ainsi enseveli en utilisant les murs arasés comme fondation. La physionomie actuelle du site est entièrement tributaire de cette modalité particulière d'abandon des espaces habités. Les fameuses «maisons » de Çatalhöyük constituent autant de scènes d'inhumation volontaire, et leur préservation souvent édifiante, le résultat paradoxal de leur destruction.

Dans ce qui suit, je commencerai par m'interroger sur le statut interprétatif des assemblages archéologiques ainsi générés. Je montrerai que leur faculté à évoquer spontanément les cadres de la vie quotidienne repose en grande partie sur l'omission d'une variable essentielle de leur existence: les intentions et pratiques culturelles à l'œuvre dans la destruction des bâtiments et, par conséquent, dans la formation du site. Au-delà du biais de représentativité qui se trouve alors introduit dans l'interprétation des ensembles exhumés, j'émettrai l'hypothèse que cette dimension cachée de l'éloquence archéologique de Çatalhöyük témoigne surtout de la capacité des habitants du Néolithique à produire des vestiges significatifs pour eux-mêmes. La notion de capsule temporelle fournira ici les clefs d'un décentrement pour réinvestir les actions préhistoriques d'une valeur authentiquement prospective. En deçà de l'expérience immédiate, les ruines de Çatalhöyük apparaissent alors moins comme des restes absolument fidèles à une réalité passée - ou figée dans le temps - que comme des productions orientées vers un futur qui leur est propre.

\section{Une étrange familiarité}

Au cours de la première moitié du septième millénaire avant notre ère, Çatalhöyük était une agglomération majeure qui s'étendait sur plus de treize hectares et réunissait une population estimée entre cinq et huit mille personnes (Düring 2006: 234-235). L'histoire du site remonte à la fin du huitième millénaire, lorsqu'il s'impose comme une centralité à l'échelle de la plaine de Konya et comme un point de convergence des réseaux d'échange qui parcourent I'Anatolie du Sud et le Levant au Néolithique (Baird 2005). Aussi importante soit-elle pour comprendre la dynamique de ce phénomène d'agrégation, cette phase initiale demeure virtuellement inexplorée. La connaissance des premiers niveaux d'occupation est en effet tributaire de sondages périlleux à travers des couches de vestiges ultérieures qui s'accumulent, par endroit, sur plus de vingt mètres d'épaisseur (Bayliss et al. 2015). Le tout forme ainsi une grande éminence artificielle: un höyük en turc (littéralement une «colline»), quoique le jargon archéologique privilégie son équivalent arabe, un tell, pour décrire cette modalité courante de la formation des sites du Proche-Orient ancien (fig. 3). Durant la seconde moitié du septième millénaire, tandis que l'établissement dominait la plaine d'une quinzaine de mètres, un ensemble de transformations sociales, économiques et idéologiques participèrent à réduire la superficie de la zone habitée, à fragmenter sa trame architecturale et à accentuer la mobilité de ses habitants, jusqu'à l'abandon définitif du site et la dispersion de sa population au tournant du sixième millénaire (Hodder 2014).

\section{ci-contre}

fig. 2

La sépulture multiple en cours de fouille du bâtiment 52. On reconnaît ici le haut du crâne et les jambes repliées de l'adulte, reposant sur le dos, ainsi que le squelette de l'enfant le plus complet. Sur ce dernier, les éléments foncés correspondent aux restes organiques: en couronne, sur sa tête, ceux d'un bol qui recouvrait initialement le côté gauche du crâne; en lambeaux sur l'abdomen, ceux de la pièce de lin qui séparait l'enfant du nourrisson placé directement au-dessus de lui. Çatalhöyük Research Project, photo Scott Haddow. 


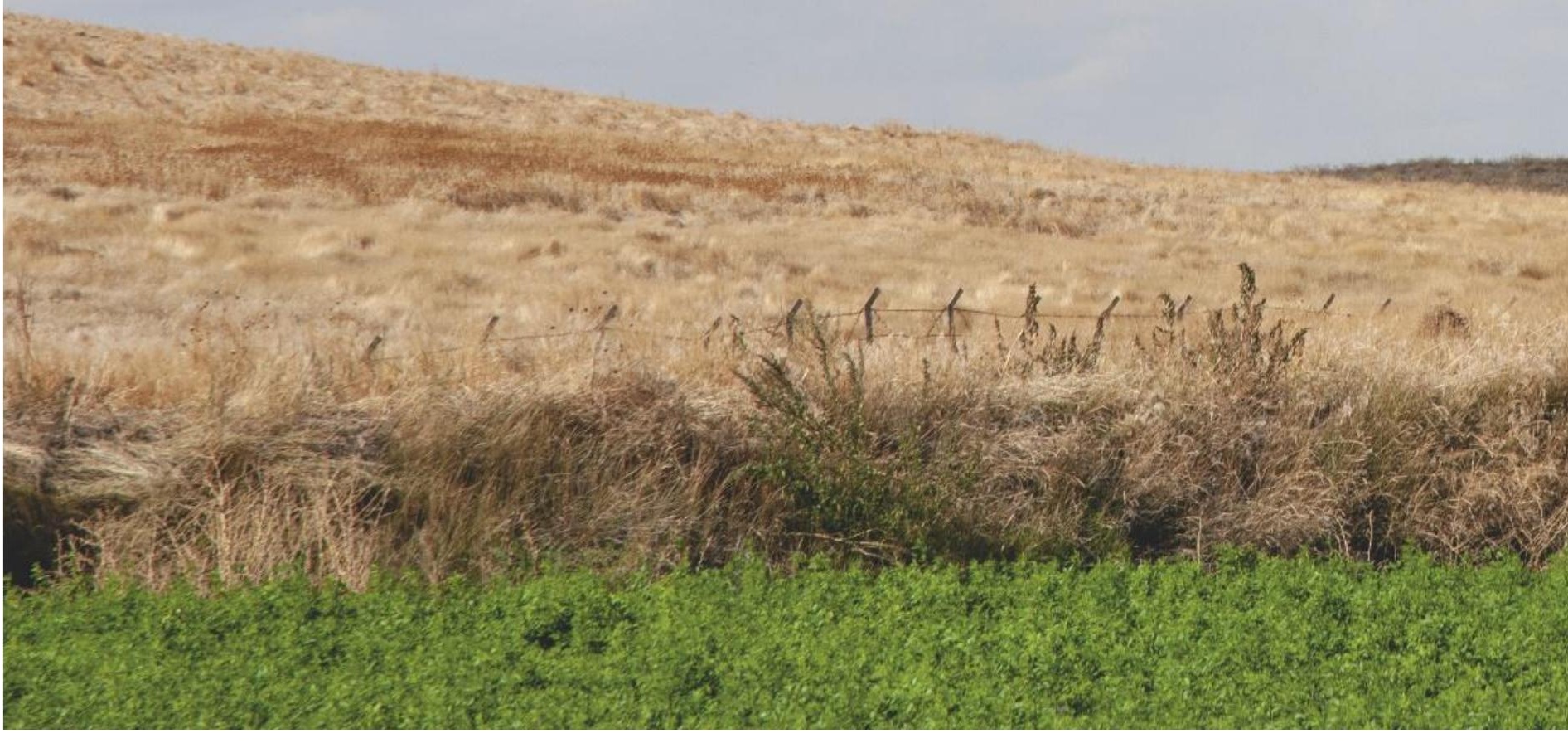




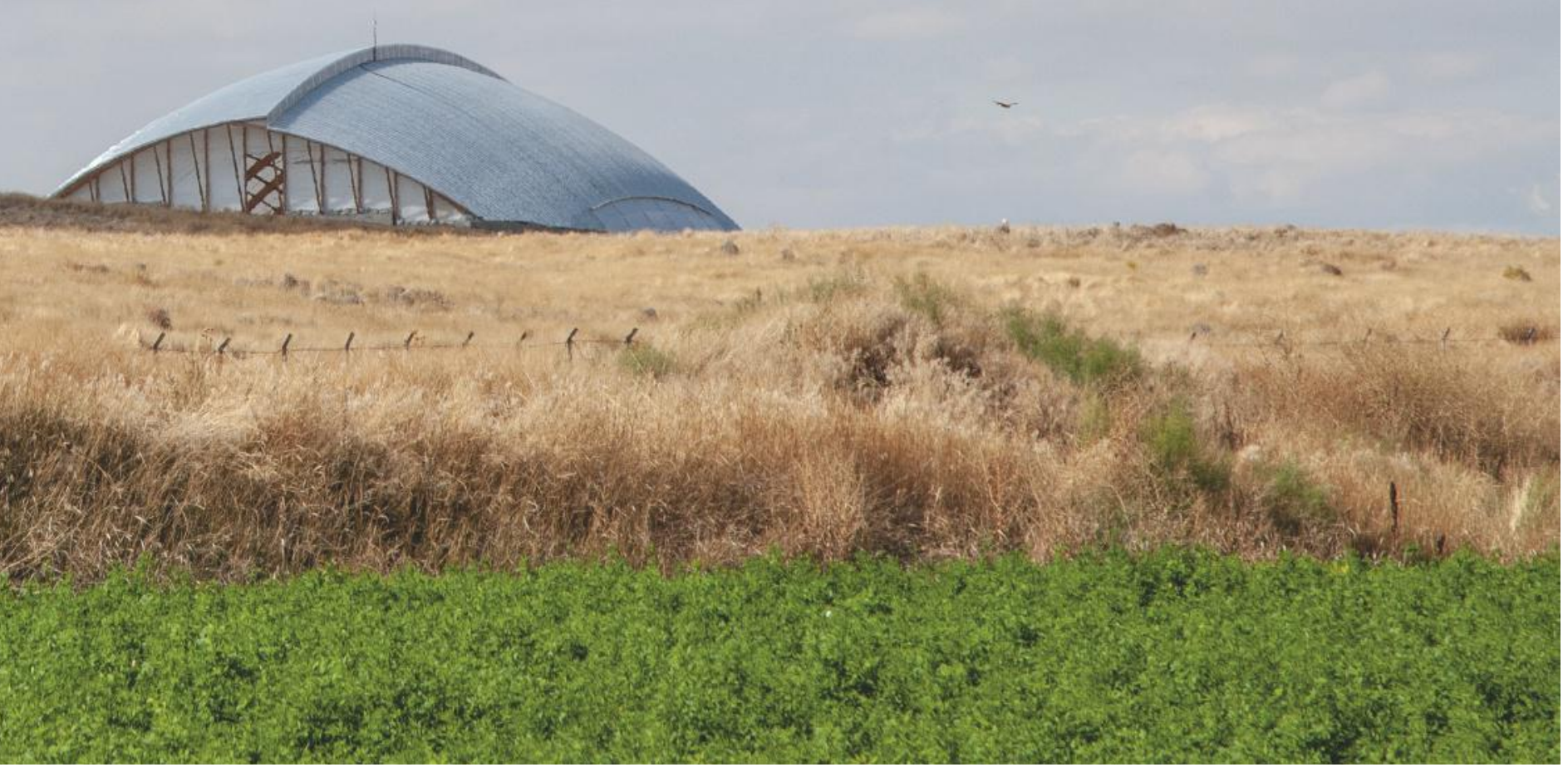


fig. 4

Plan du tell de Çatalhöyük, avec les deux principaux secteurs de fouille, et le détail du niveau VIb dégagé par Mellaart au sud dans les années 1960 (d'après Mellaart 1967 et Hodder 2014).
2. II s'agit, plus précisément, de briques de terre crue, aussi larges que le mur, appareillé longitudinalement avec un épais mortier sableux et calcaire, et recouvert de couches successives d'enduit composé d'argile et de chaux.

\section{La compilation} systématique des données quantitatives sur l'architecture a permis d'obtenir, pour la superficie intérieure des bâtiments, une répartition quasi gaussienne entre dix et cinquante mètres carrés avec un pic très accentué entre vingt-cing et trente mètres carrés (Düring 2007 : 163). La variation s'explique davantage par le caractère modulaire de l'architecture (c'est-à-dire le nombre de pièces subsidiaires)

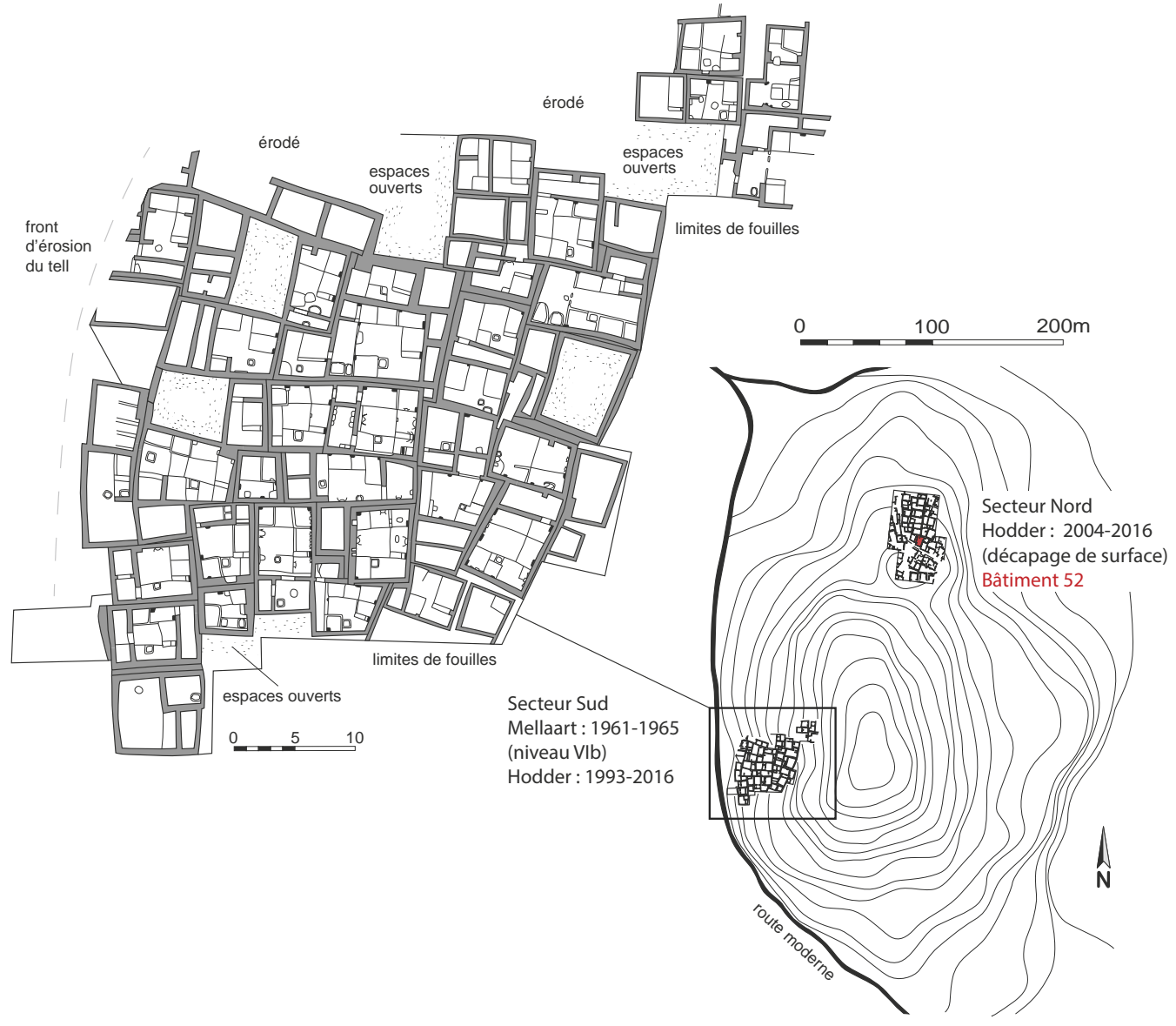

que par la taille respective des espaces (relativement constante). La corrélation est d'ailleurs très faible entre ces deux facteurs (Hodder et Pels 2010: 173), ce qui renforce d'autant l'impression générale d'uniformité. Certains bâtiments concentrent certes les sépultures, d'autres des attributs symboliques (bucranes, peintures, etc.), mais ces variables ne se recoupent pas systématiquement, pas plus qu'elles ne recoupent celle de la taille (ibid.). Aucun seuil objectif ne peut donc être établi pour distinguer parmi les bâtiments ce que James Mellaart désignait arbitrairement comme des «sanctuaires».
Accessibles sur de larges portions du tell, en partie découverts par l'érosion, les niveaux intermédiaires sont donc les mieux connus (VII-VI dans la chronologie de Mellaart; L-O South et F-G North dans celle d'Hodder, vers $6800-6400)$. Ils sont parfois qualifiés de «classiques » du fait de la place qu'ils occupent dans l'image archétypale véhiculée par l'abondante littérature, aussi bien archéologique que grand public, sur Çatalhöyük. Leur fouille extensive a livré un paysage bâti saisissant, à l'allure quasi urbaine, qui a beaucoup contribué à la notoriété du site (fig. 4). Les murs en adobe ${ }^{2}$ conservés sur des hauteurs conséquentes - rarement moins d'un mètre, parfois jusqu'à deux dessinent un plan d'une grande densité. Les bâtiments sont en effet tous accolés les uns aux autres selon une forme dite «agglutinante », sans espaces interstitiels hormis quelques emplacements servant momentanément de dépotoirs et d'étroites «allées» vides qui semblent délimiter de grands ensembles de plusieurs dizaines d'unités sans toutefois servir de voies de communication. Le degré de standardisation formelle des bâtiments est très élevé: tous déclinent un plan rectangulaire analogue et plus ou moins compartimenté entre une pièce principale et des espaces périphériques (souvent dédiés au stockage ou aux activités culinaires), leur superficie moyenne ne variant que de façon relative ${ }^{3}$. Contrairement à l'idée que l'on se fait généralement d'une ville, toutefois, aucune structure ne se démarque par sa fonction ou sa monumentalité dans les zones prospectées. Aucune place, aucune rue véritable, ne vient rompre cette répétition d'unités architecturales juxtaposées. 
En l'absence de portes communiquant entre bâtiments ou vers l'extérieur, les habitants sont supposés circuler sur des toits-terrasses où se situaient les seuls accès aux pièces intérieures. Le statut de ces espaces sommitaux, que l'on devait nécessairement traverser pour accéder d'un bâtiment à un autre, et où devait néanmoins se dérouler une partie des activités domestiques, interroge. L'invisibilité archéologique de ces «toits», qui font pourtant le propre de la vie sociale et de l'expérience architecturale à Çatalhöyük, contraste avec l'excellente conservation des pièces principales qui donnent, à elles seules, l'impression d'un aperçu fidèle et représentatif de la vie néolithique. Celles-ci sont en effet divisées entre un côté sud par lequel on pénètre et où se trouvent des équipements «utilitaires", comme les fours ou les foyers, et un côté nord qui concentre les attributs les plus marqués «symboliquement »: tombes, peintures murales et autres reliques ou trophées, comme les crânes et massacres d'aurochs intégrés à la matière même du bâti par le façonnage de réceptacles en terre (fig. 5). À la complémentarité difficile à concevoir à travers notre prisme culturel entre l'espace extérieur des toits, à la fois dépendant et ouvert, et le domaine intérieur des pièces, investi autant par des activités ménagères que rituelles, se substitue ainsi dans chaque bâtiment une polarité réductrice mais autrement plus intuitive.

La force visuelle de ces pièces a attisé bien des curiosités. Parmi les préhistoriens, Çatalhöyük (du moins dans sa phase «classique») a souvent été considérée à la fois comme l'héritière et l'apogée des cultures du Néolithique levantin, au point de souvent servir d'entrée pour une réinterprétation générale du processus de domestication (Mellaart 1975; Hodder 1990; Cauvin 1997). Mais le pittoresque du site conjugué à son ancienneté lui réserve également une place de choix dans les tentatives les plus diverses de dégager une vérité universelle sur l'évolution humaine (Lewis-William et Pearce 2005; Testart 2010; Girard 2015). Indépendamment de leurs qualités et défauts réciproques, ces lectures partagent toutes une même fascination pour l'association en un même lieu des indices familiers d'une vie domestique avec ceux, plus étranges, d'une cosmologie exubérante et picturale. Les figures humaines qui, sur certaines peintures murales, sont représentées en relation avec des animaux aux dimensions parfois démesurées, ont tout particulièrement focalisé le regard: la fresque des vautours qui semblent s'attaquer à des corps acéphales (que certains voudraient voir décharnés dans le cadre d'un rite funéraire ou d'une chasse aux têtes) et celle de l'aurochs encerclé par une foule dans ce qui ressemble à une chasse collective (ou, pour beaucoup, un «sacrifice») sont régulièrement présentées comme des projections littérales de la vie au Néolithique.

Si l'on ajoute à cela la présence physique des morts dans l'architecture, Çatalhöyük procure le sentiment troublant d'une grande proximité avec les personnes qui y ont vécu. «C'est comme si le sens donné à la vie sociale était tout entier contenu dans les maisons », a ainsi pu résumer un anthropologue récemment invité à contribuer à l'interprétation du site (Bloch 2010: 150). Dès l'introduction à son ouvrage de synthèse sur les résultats des fouilles récentes, lan Hodder s'étend sur ce phénomène qui «donne au visiteur l'impression de pouvoir presque toucher le passé, de voyager dans le temps [...] pour se retrouver face à son ancêtre d'il y a 376 générations ". «C'est cela, ajoute-t-il, qui draine tant d'attention sur un site pourtant si étrange, si incongru, voire franchement inquiétant ${ }^{4}$.» (Hodder 2006: 23-24)
4. Cette traduction et toute celles qui suivent sont de l'auteur.

\author{
double page \\ précédente \\ fig. 3 \\ Une portion du tell \\ de Çatalhöyük et l'abri \\ protégeant le secteur de \\ fouille nord. Turquie, 2009. \\ Photo (C) Jason Quinlan.
}




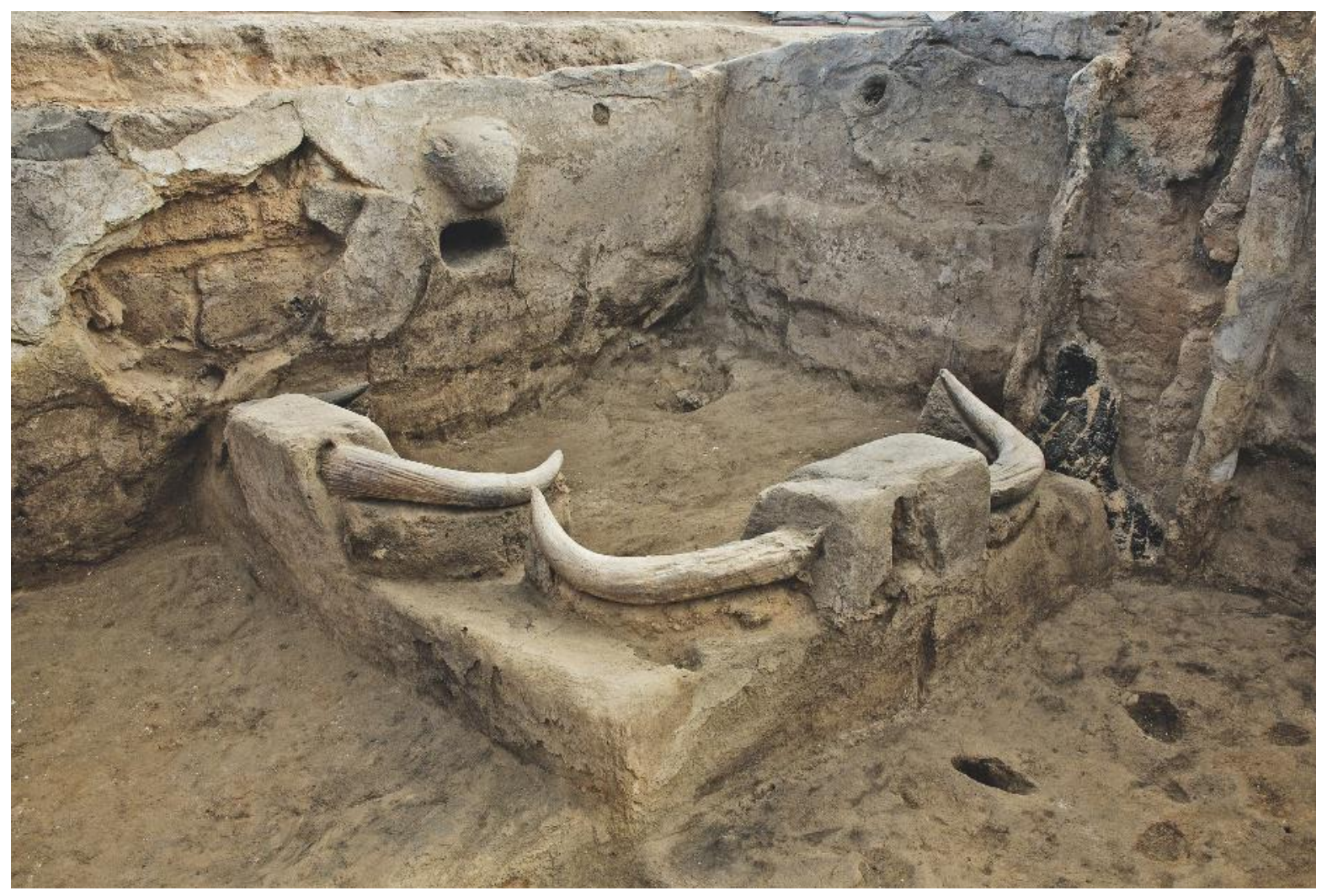

fig. 5

La "banquette" aux aurochs.

Plateforme funéraire dans le coin nord-est

du bâtiment 77 (incendié, comme le 52,

avant son comblement). On distingue à droite

les restes carbonisés d'un pilier en bois.

À gauche, l'ébréchure dans le mur laisse

entrevoir la structure en adobe et l'épaisseur des couches d'enduit superposées. À côté,

au-dessus de la petite niche, la protubérance contient le crâne d'un veau dont elle remodèle la tête (les cornes ont été détruites par l'incendie ou brisées avant le comblement). Une fois que les couches supérieures d'enduit seront retirées par les fouilleurs, une peinture rouge vif apparaîtra sur tous ces réceptacles de massacres bovins, la niche et la plateforme, ainsi qu'une série de mains positives,

de la même couleur, plus haut sur le mur.

Turquie, 2008. Photo @ Jason Quinlan. 
Dans sa préface à la monographie de Mellaart, le célèbre archéologue Mortimer Wheeler capturait déjà l'ambivalence qui émane de ces bâtiments, partagé entre la facilité à s'y projeter et le caractère «souvent bizarre et un brin morbide» de leur symbolisme, entre la transparence formelle des ruines et «la communauté ésotérique» qui les a peuplées (Mellaart 1967: 10).

D'une manière générale, les vestiges archéologiques, les restes humains et l'acte même d'exhumer sont propices à cette sensation d'inquiétante étrangeté ou, plus exactement, d'Unheimliche pour reprendre le substantif intraduisible rendu célèbre par Sigmund Freud (1985). Les réactions équivoques des Modernes face à ce type d'atmosphère ont joué un rôle majeur dans l'émergence d'une sensibilité archéologique au XIX siècle, ainsi qu'en témoigne, par exemple, la fascination exercée très tôt par Pompéi (Vidler 1992; Freud 2013). Mais la source de cette étrangeté ne se limite pas à l'irruption, dans un cadre domestique (heimlich), d'un élément antithétique (un-), comme cela a pu être récemment proposé pour la polarité des espaces intérieurs à Çatalhöyük (Weismantel 2014) ${ }^{5}$. Cette sensation tient moins à «une religiosité et une esthétique intrinsèquement étrangères au tempérament moderne» qu'aux «proportions intimistes [des espaces] et [aux] traces d'une vie somme toute très banale» (ibid.: 262). Le fait que Çatalhöyük demeure intelligible en dépit du caractère très déroutant de ses vestiges est précisément ce qui permet à l'Unheimliche de s'installer au sens - plus strictement freudien - de ce qui est secrètement trop familier (heimisch) ${ }^{6}$. Ce que Freud qualifie de «retour non intentionnel du même» passe ici par un processus d'identification renforcé par la proximité des morts, par une capacité spontanée à peupler l'espace de sa propre expérience et d'en apprécier, dans le même temps, tous les décalages. Les objets en apparence les plus triviaux participent pleinement à cette ambiance précisément parce qu'ils conjuguent l'assimilable et l'étrange. L'Unheimliche culmine sans aucun doute dans les situations les plus ambiguës: avec le reflet d'un fouilleur dans un miroir d'obsidienne, l'exhumation d'un crâne surmodelé ou, plus prosaïquement, une trace de main dans la terre à bâtir.

Ce sentiment de présence/absence est normalement plus commun dans l'archéologie des périodes contemporaines, dont les vestiges sont plus directement identifiables, que dans des contextes anciens marqués par une forte altérité culturelle, où le travail d'interprétation s'apparente davantage à «une stratégie de domestication [...] pour familiariser ce qui est intrinsèquement étranger» (Buchli et Lucas 2001: 9-12). Si donc un cas aussi lointain que Çatalhöyük produit le même type d'assimilations spontanées que les restes d'un passé récent, c'est à la différence toutefois que celles-ci ne reposent plus sur une proximité altérée, mais sur une familiarité reconstituée par un regard actuel. D'où le risque d'un exercice analogique répondant à des catégories descriptives inadéquates et donnant prématurément la sensation de comprendre. L'illustration la plus évidente d'une telle tendance réside peutêtre dans la notion de «maison » utilisée sans autres précisions pour désigner des bâtiments dont on peine à saisir le statut exact, où se mêlent activités résidentielles, cérémonielles, funéraires et artisanales, et où s'interpénètrent sphères «publique» et «domestique» (si tant est que de telles catégories aient ici un sens).
5. Cette position est en fait plus fidèle à la théorisation originale de l'Unheimliche par Ernst Jentsch que Sigmund Freud s'applique précisément à dépasser dans son essai.

6. [Ce qui est secret ou caché] est le sens second (quoique plus courant en allemand moderne) de l'adjectif heimlich [étymologiquement: "domestique»] ici substantivé. La métaphore archéologique de l'exhumation, comme toujours chez Sigmund Freud, est explicite.

C'est ce qui lui permet d'interpréter l'Unheimliche comme le symptôme du refoulement de l'Heimliche-Heimische (d'un familier déjà présent, bien que caché, donc: le préfixe marquant le refus et non la négation). Mais Freud donne lui-même les clefs d'un dépassement de cette perspective clinique en fondant son raisonnement sur l'analyse de textes littéraires et en annonçant s'attaquer à un problème d'esthétique générale qui dépasse le cadre biographique ou celui de la cure psychanalytique. À cet égard, la pertinence du concept est appréciable du fait que ce n'est évidemment pas dans les récits les plus baroques ou fantaisistes que l'on fait l'expérience de "l'inquiétante étrangeté", mais bel et bien dans l'intrusion du fantastique dans un réel familier. En lui-même, l'étrange est un antidote à l'Unheimliche. D'où les problèmes maintes fois soulignés de la traduction française de ce terme. 
En bref, l'expérience archéologique de Çatalhöyük est conditionnée par l'attrait, à la fois intellectuel et esthétique, qu'exercent ces pièces encastrées aux allures de diorama, où toutes les dimensions rituelles et domestiques de la vie sociale semblent représentées de façon cohérente au point d'en faire oublier le caractère partiel à l'échelle du site. Mais les implications interprétatives de cette fascination dépassent la seule réduction du regard porté rétrospectivement sur l'établissement néolithique. Elles constituent un obstacle épistémologique au sens strict où Gaston Bachelard l'entendait, une «idée scientifique trop familière [qui] se charge d'un concret psychologique trop lourd, [qui] amasse trop d'analogies, d'images, de métaphores, et [qui] perd peu à peu son vecteur d'abstraction» (Bachelard 1938: 15). En l'occurrence, je voudrais désormais montrer en quoi la puissance d'évocation de ces vestiges altère paradoxalement leur portée archéologique plus abstraite, c'est-à-dire leur capacité à informer des actions, des normes et des représentations de la société qui habitait Çatalhöyük au Néolithique.

\section{Un Pompéi néolithique?}

S'agissant de «reconstruire la vie préhistorique», la notion de sol d'habitat a fait l'objet d'un fétichisme certes compréhensible mais souvent préjudiciable (Bordes 1975). Les surfaces décapées où se dessinent les restes présumés structurés d'une occupation ont en effet pour elles une qualité visuelle quasi photographique. S'il est tentant d'y voir autant d'instantanés de l'occupation à un temps $\mathrm{T}$, ceci ne vaut qu'à condition qu'elles couvrent un «laps de temps suffisamment court pour que l'on puisse espérer déduire de la position des vestiges quelque chose au sujet des activités [préhistoriques] » (ibid. : 139). Dans les faits, cette condition préalable n'est remplie que par une poignée de contextes archéologiques où un enfouissement rapide est venu figer, sans la dénaturer, une distribution encore signifiante des restes de l'occupation. D'où l'importance, surtout pour les périodes les plus anciennes, de la taphonomie, c'est-à-dire l'étude des dynamiques d'inhumation (en grec, taphos) et de leurs influences (perturbatrices ou préservatrices) sur les vestiges ainsi constitués. II devient alors possible d'écarter toute interprétation précipitée comme celles fondées, par exemple, sur des palimpsestes ou des assemblages faussés par les processus post-dépositionnels, voire, dans le meilleur des cas, de corriger les défauts pour isoler dans le désordre initial d'une couche archéologique les relations restées pertinentes.

À Çatalhöyük, ce débat prend une tournure singulière. D'un côté, il est relativement facile de suivre, l'un après l'autre, les sols superposés. Chaque couche d'enduits correspondant à une réfection saisonnière, une modification architecturale ou l'inhumation d'un défunt, les décapages successifs révèlent des états parfaitement scellés et conservés du bâtiment - mis à part d'éventuelles démolitions. De l'autre, on n'y retrouve généralement pas ou peu d'objets qui définiraient ailleurs un épisode d'occupation. Ce qui frappe d'entrée à Çatalhöyük, c'est la propreté des «maisons»: il n'y a pas grandchose sur ces sols pour habiller - ou contredire - l'éloquence du cadre architectural. Aussi, lorsque certains de ces bâtiments livrent des objets sur leur dernier sol, «a red flag goes up! » comme le dit Ruth Tringham (2013: 97). 
Au moment de l'abandon définitif d'un bâtiment, nul besoin en effet de nettoyer aussi consciencieusement les sols que lors d'une réfection. Les pratiques de destruction qui accompagnent la fin d'une occupation, on l'a vu, condamnent et protègent le lieu en l'enfouissant sous une épaisse couche de remblais. À aucun moment l'espace n'est donc exposé aux facteurs taphonomiques - climatiques, environnementaux ou humains - à même de perturber la disposition post-dépositionnelle des artefacts. Ces assemblages sont donc ce qui se rapproche le plus, à Çatalhöyük, d'un sol d'habitat (fig. 6). D'autant que les réfections et le nettoyage régulier des surfaces réfutent l'hypothèse d'un palimpseste. II paraît alors logique de chercher dans ces restes des traces d'activités in situ. La distribution des ressources animales et végétales sur les derniers sols du bâtiment 52 a ainsi pu être utilisée pour caractériser l'économie domestique et la fonction spécialisée des différentes parties de l'espace habité (Bogaard et al. 2009; Twiss et al. 2009). Ailleurs, la répartition différentielle du gros outillage lithique de mouture, de broyage ou de percussion entre des bâtiments contemporains a quant à elle été mobilisée pour identifier une «inégalité sociale émergente entre «maisonnées» (Wright 2014).

L'absence de perturbations post-dépositionnelles n'équivaut pourtant pas à l'absence de biais taphonomiques: encore faut-il, en effet, s'assurer que les dynamiques dépositionnelles soient bien conformes aux pratiques qu'on leur assigne. Ces analyses acceptent toutes comme prémisse que le comblement de la pièce a figé les objets dans une position représentative de la période d'occupation. Qu'elle soit vraie ou pas des assemblages en question ${ }^{7}$, cette proposition n'est jamais vraiment démontrée. Son unique véritable épreuve réside tautologiquement dans la cohérence des résultats obtenus. Or il suffirait que certains espaces aient été partiellement nettoyés ou qu'un objet ait été ajouté au cours de la procédure de condamnation pour que l'interprétation générale des résultats en soit faussée.

Dans un cas particulier, cependant, cette question de la représentativité est explicitement posée. La controverse sur la nature intentionnelle ou accidentelle des incendies comme celui du bâtiment 52 naît en partie de l'espoir d'identifier des dépôts représentatifs d'activités domestiques. La thèse de l'accident permettrait en effet d'envisager les restes comme un état figé de l'occupation. Mellaart imagina ainsi que son niveau IVa, où se concentrait en effet une grande partie des bâtiments brûlés au début de la fouille, eût pu être détruit par un unique et désastreux épisode (Mellaart 1964). Mais il remarqua lui-même par la suite que de tels contextes étaient également répandus dans d'autres niveaux, avec une fréquence renforcée à mesure que l'on avançait dans I'histoire du site. À la lumière des fouilles actuelles et d'analyses plus précises des modalités techniques d'allumage et de propagation du feu dans ce type d'architecture fermée en terre, il apparaît désormais de plus en plus certain que ces incendies ne se transmettaient pas d'un bâtiment à un autre, mais nécessitaient à chaque fois d'accumuler du combustible ou d'aménager un apport en air dans la toiture, et dénotent donc, globalement, une certaine maîtrise de l'événement (Cessford et Near 2005; Harrison et al. 2013; Tringham 2013). La contemporanéité de ces épisodes avec des dépôts singuliers, comme la sépulture multiple du bâtiment 52 , suggère des formes ritualisées de destruction dont on connaît des antécédents spectaculaires dans le Précéramique levantin (Hadad 2019)
7. Cela a sans doute d'autant plus de chances d'être vrai qu'il s'agit de micro-restes (mais voir Cessford 2003). Même dans ce cas, il faut accepter au préalable que l'image spatiale qui se dégage de l'analyse n'est alors plus la visualisation d'un temps $T$ mais un palimpseste dont il faut postuler - encore une fois - qu'il est représentatif d'une structuration valable pour toute la durée d'occupation de la surface. 


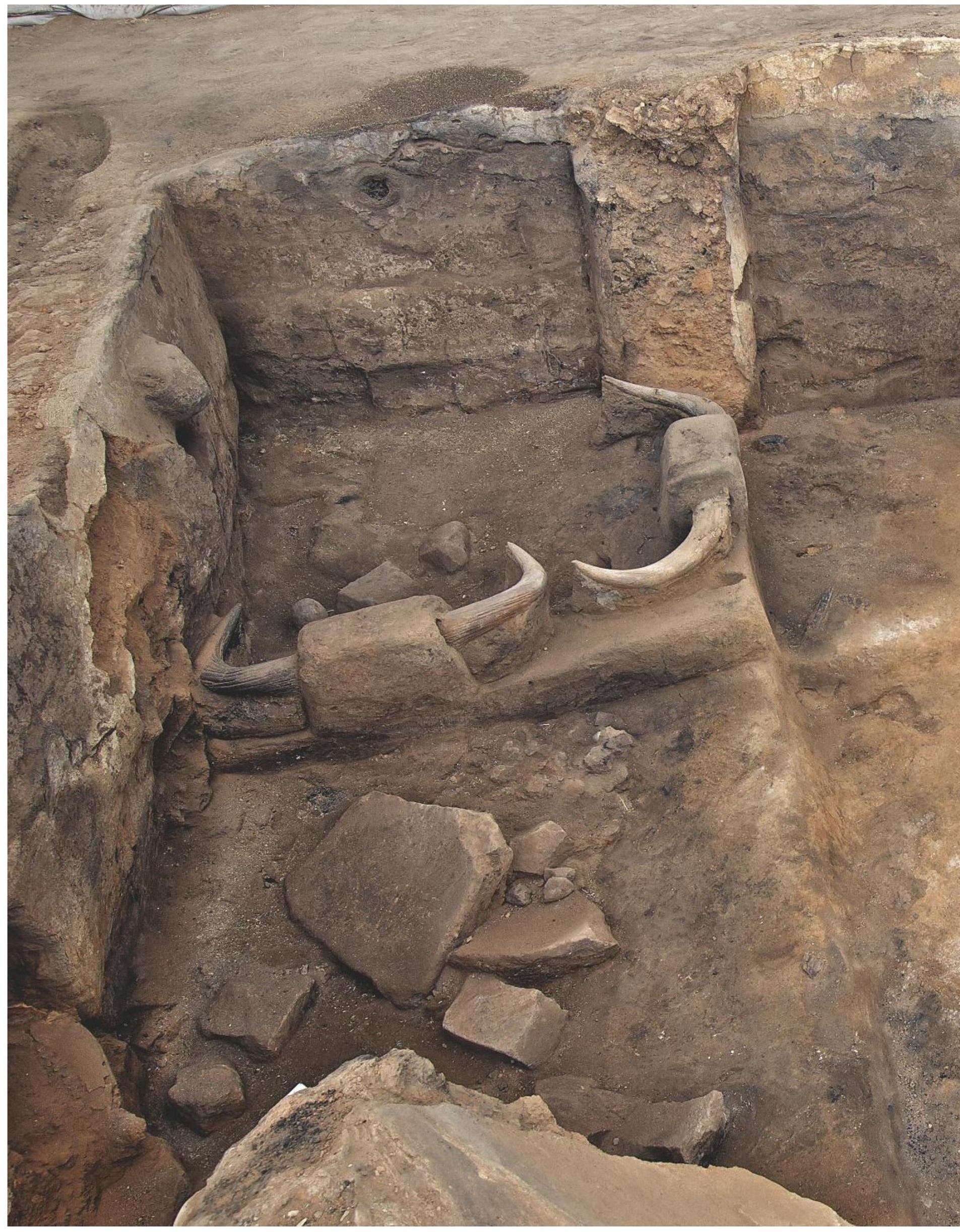




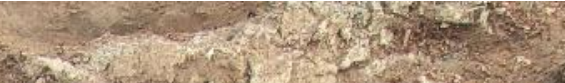

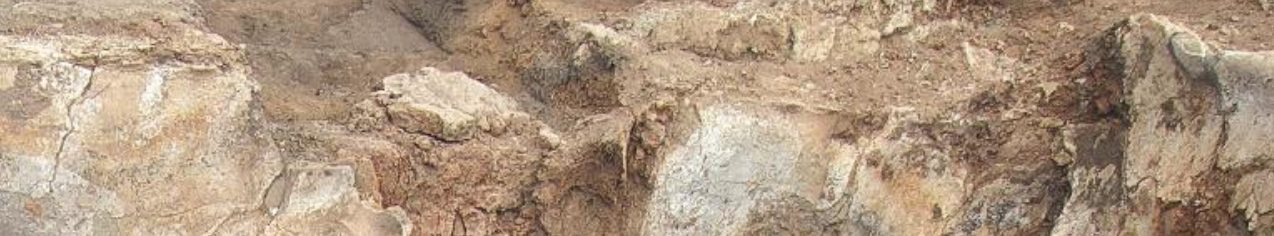

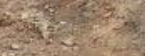

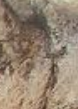

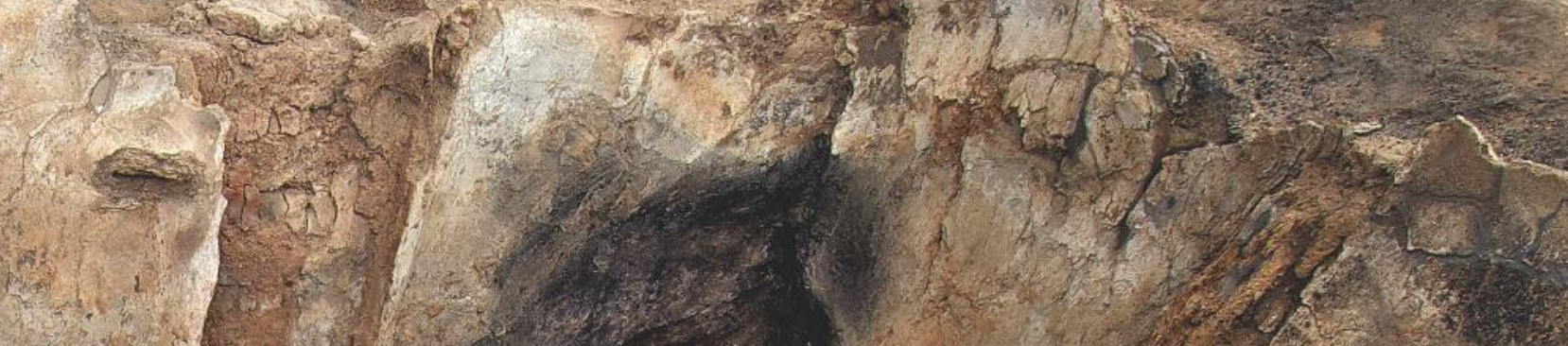

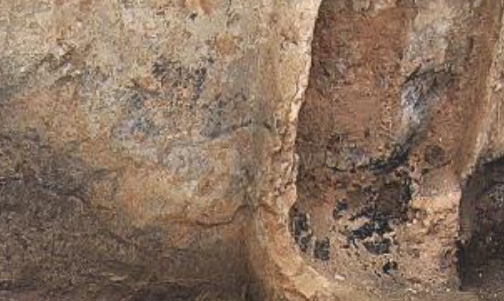
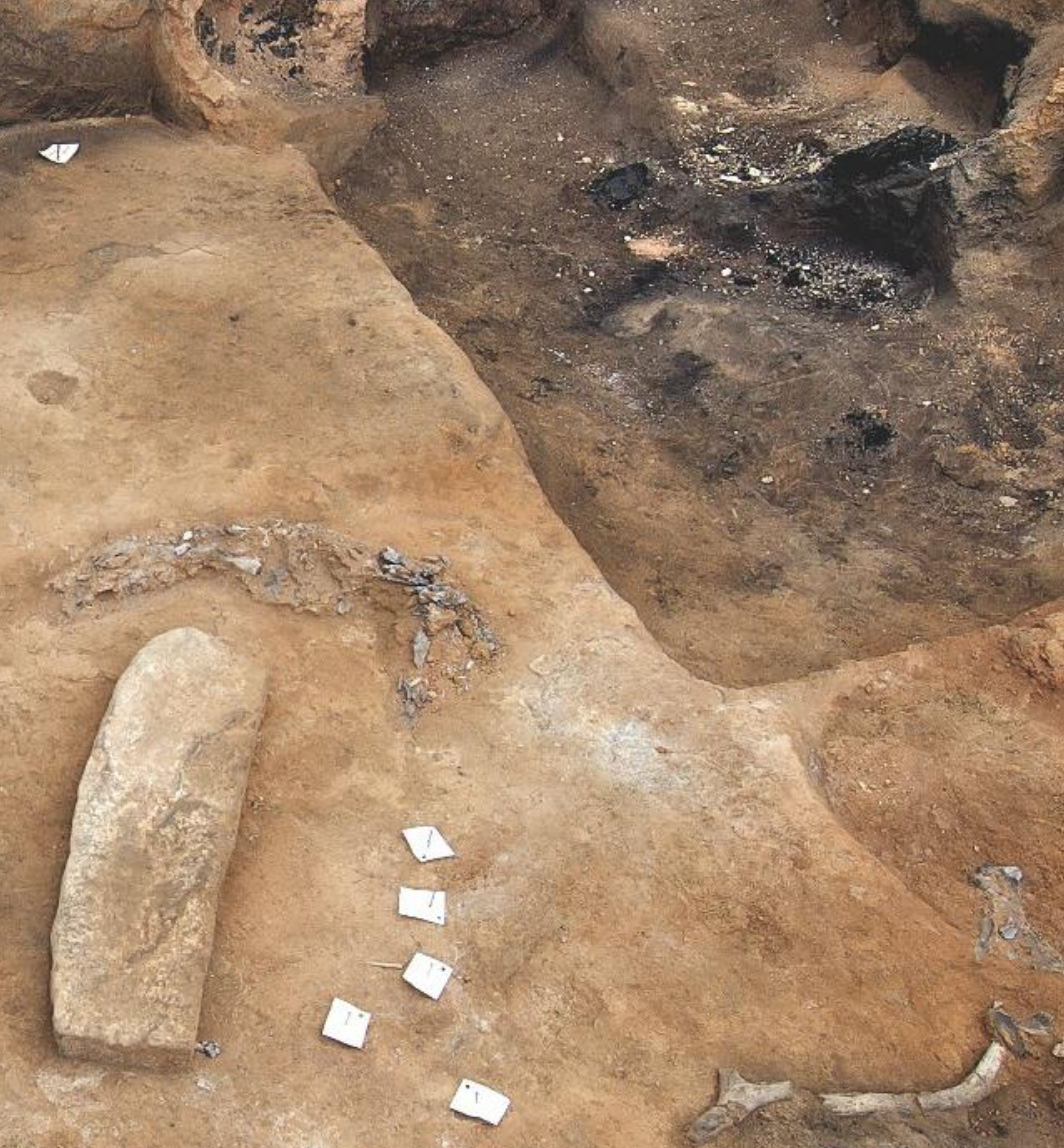

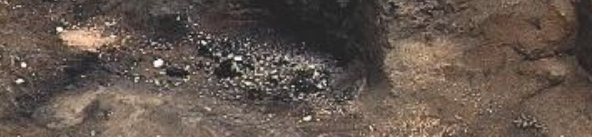
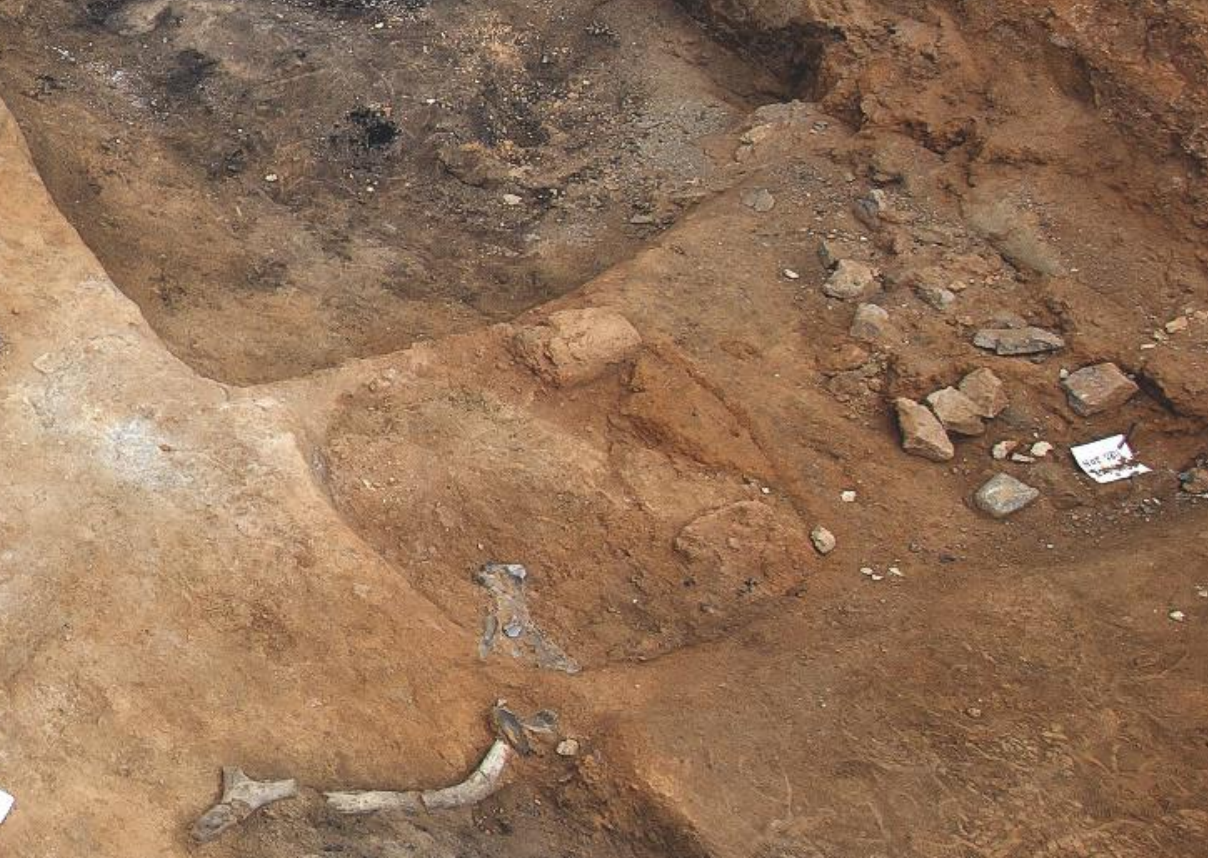

$x^{2}+x^{2}+2$

a 2

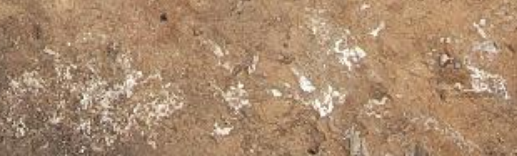

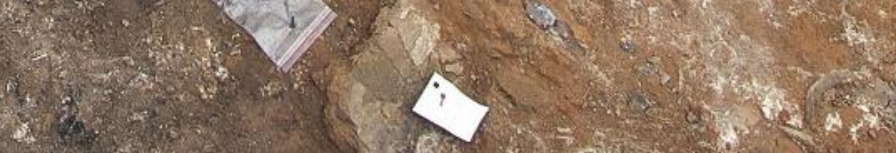




\section{double page précédente}

fig. 6

Divers vestiges et artefacts

(une hache ou une meule

sur le sol) sur le dernier

«sol» d'occupation du

bâtiment 77 , contemporain

de l'incendie et du

comblement, 2008

Photo (C) Jason Quinlan. et qui sont appelées à se répandre dans l'architecture domestique de certains contextes néolithiques plus tardifs du Proche-Orient (Verhoeven 2000), de Chypre (Le Brun 2003) ou des Balkans (Stevanović 1997; Tringham 2000).

La thèse de l'accident inopiné ne s'appuie en fait que sur le seul argument de la présence d'éléments architecturaux précieux (des poutres laissées en place, les crânes d'aurochs...), de ressources (du grain dans les silos, des "caches» d'obsidienne....) et d'artefacts (une hache ou une meule sur le sol...) dans l'espace abandonné. Non seulement cela ne réfute en rien I'hypothèse d'une destruction volontaire et planifiée, puisque la perte de ces objets s'intègre potentiellement à la logique dispendieuse d'une condamnation ritualisée (Russell et al. 2014), mais ce raisonnement repose, ici encore, sur une logique circulaire où ce qu'il propose d'inférer (les activités reflétées par les assemblages) sert également de prémisse (les assemblages reflétés par les activités). Si la thèse de l'accident n'est pas systématiquement à exclure, elle nécessite donc d'être démontrée au cas par cas par des arguments positifs. Or elle est généralement considérée par défaut: là où l'intention fait sans cesse l'objet d'un doute, la fidélité des horizons de destructions à un état figé de la vie des habitants s'impose comme une évidence.

L'interprétation scientifique rejoint ici l'esthétique unheimliche qui façonne l'expérience contemporaine du site. La profusion d'éléments conduit en effet à ce que Roland Barthes a appelé dans un contexte littéraire un « effet de réel». De la même manière que les "détails inutiles" sont au service du réalisme d'un texte par le biais d'une «illusion référentielle» (Barthes 1968: 88), l'abondance et la bonne conservation des vestiges alimentent artificiellement la vraisemblance des scénarios qui reposent davantage sur leur intelligibilité spontanée que sur une mise en contexte potentiellement contre-intuitive. Le caractère a priori évident du contexte a ainsi tendance à orienter, au prisme des attentes et de l'imaginaire anthropologique, l'interprétation vers une représentation générique, familière, de la vie préhistorique. Cette illusion du vraisemblable incite alors à ignorer des discordances d'autant plus nombreuses que le site est riche et complexe, pour se concentrer sur ce que l'on pourrait appeler une histoire immédiate et intuitive. C'est là le véritable paradoxe de la préservation des espaces détruits: l'impression qu'elle génère est un obstacle pour penser les pratiques qu'elle recouvre.

Cet écueil fait écho à ce que Robert Ascher a qualifié de «prémisse de Pompéi» pour critiquer l'impression que la fouille viendrait «perturber les restes d'une communauté, qui fut vivante, avant de s'arrêter, comme figée, à un temps T» (Ascher 1961: 324). Pour Ascher, au contraire, «l'archéologue ne fait qu'interrompre un processus de décomposition » entamé - bien avant l'état de vestige - par «le changement perpétuel qui caractérise chaque communauté vivante» (ibid. ; Ascher 1968). Ici, privée d'un point de référence arrêté, l'illusion de la représentativité présumée des assemblages n'est donc plus permise. Mais cette définition initiale de la «prémisse de Pompéi » est souvent éclipsée par la dispute qui opposa à son propos deux grandes figures de l'archéologie américaine. Du biais interprétatif en question, ils ne retinrent en effet chacun qu'une conception restrictive leur permettant de se renvoyer mutuellement l'anathème.

D'un côté, Michael Schiffer promut ce qui deviendra l'approche taphonomique classique: afin de ne pas prendre les sites archéologiques pour les Pompéi qu'ils ne sont pas, il convient de considérer en priorité l'ensemble 
des processus - dépositionnels et post-dépositionels, culturels ou environnementaux - à l'œuvre dans la formation des vestiges (Schiffer 1985). Autrement dit, si l'objectif est bien de documenter les sociétés passées, l'archéologue doit d'abord s'attacher à comprendre les manières dont les vestiges ont été «déformés». Lewis Binford (1981), pour sa part, considéra qu'une telle approche reposait sur une analogie trompeuse avec le présent ethnographique. En postulant l'existence d'un état initial à purger de toutes altérations ultérieures, et donc des scènes de vie idéales à «reconstruire», elle considère tous les sites comme des Pompéi en puissance. Or les données archéologiques n'ont nul besoin de se conformer à un tel idéal pour être signifiantes. «Elles ne peuvent être considérées comme déformées qu'en fonction d'attentes définies a priori, mais certainement pas en fonction de leur réalité propre." (ibid.: 200) Ce raisonnement renoue potentiellement avec le dynamisme intrinsèque qu'Ascher reconnaissait aux vestiges pour les affranchir de l'illusion référentielle, mais il n'est pas suivi jusqu'au bout. Car à défaut de «réalité propre», les vestiges demeurent pour Binford les médiateurs statiques vers un niveau de réalité passé et supérieur. II n'échappe en effet à l'image des scènes de vie à reconstruire qu'en la remplaçant par celle de la «fossilisation» de "systèmes culturels» déconnectés de la matérialité archéologique. Dans ce modèle, il n'y a de place pour les vestiges que comme des produits secondaires et passifs des occupations. Si Binford insiste avec raison sur le fait que les processus taphonomiques ne constituent pas des biais à neutraliser mais font partie intégrante du système culturel, il reste donc aveugle à ce qu'ils permettent d'inférer de comportements sociaux par leur participation à la formation des sites.

À Çatalhöyük, les pratiques de réfection et de condamnation font du site le produit d'un processus unique de formation où les logiques dépositionnelles annulent l'influence des processus post-dépositionnels. La «prémisse de Pompéi " s'y exprime donc avec une force que ni l'image de la déformation ni celle de la fossilisation n'arrivent à conjurer. Schiffer et Binford éclairent le problème depuis des perspectives plus complémentaires qu'ils ne veulent bien l'admettre: en plaçant la conservation différentielle et les processus de formation du site au centre du raisonnement archéologique, pour le premier, ou en condamnant le mirage ethnographique qui anime une conception strictement corrective de la taphonomie, pour le second. Mais ils posent tous deux la question de la représentativité des vestiges dans les termes traditionnels du débat taphonomique sur les sols d'habitat: par la «reconstruction» de différents moments de l'habitation pour Schiffer, par leur «réfléchissement » dans une temporalité palimpsestique pour Binford. En gardant l'occupation comme unique horizon interprétatif, les deux perspectives accréditent l'idée de contextes inopinément scellés et alimentent ainsi, malgré elles, l'illusion du vraisemblable.

Comme la Gradiva pompéienne de Jensen, les «maisons» de Çatalhöyük donnent l'impression de pouvoir revivre parce qu'elles ont été prises «sur le vif » (Freud 2013: 55); mais elles ne sont pas figées dans ce mouvement, elles en sont l'expression. Les réponses traditionnelles à la prémisse de Pompéi échouent à rendre compte d'un site comme Çatalhöyük car elles font l'hypothèse d'une représentativité indexée à un temps qui y fait défaut et qui, pourtant, était l'objet de la critique initiale d'Ascher. Elles passent ainsi à côté du fait que ces vestiges racontent, avant toute chose, l'histoire de leur destruction. 
8. L'exhumation programmée d'une capsule temporelle génère bien moins d'intérêt que son inhumation, sans doute parce que le souvenir de ce qui y est préservé est encore vif. Généralement fixées à des intervalles arbitraires, ces dates sont d'ailleurs suffisamment éloignées dans le futur pour que cette question importe peu dans leur conception. Comme tout autre vestige archéologique, la destinée d'une capsule temporelle est bien l'oubli, la destruction... ou sa découverte fortuite par un fouilleur, ce qui ne manquera pas de transformer son statut interprétatif: comment reconnaître un message dont on ne se sait pas destinataire?
Cela ne veut évidemment pas dire qu'ils ne témoignent jamais des activités ayant eu lieu dans l'espace concerné, mais ils ne le font qu'à la condition nécessaire d'éliminer au préalable le facteur d'ensevelissement. L'autre solution consiste à prendre ce facteur prévalent pour ce qu'il a à dire. Au-delà du fait qu'elles participent simultanément à leur préservation et à leur perturbation, il reste donc à comprendre de quelle manière et dans quelle mesure les pratiques de condamnation déterminent la production des vestiges.

\section{Çatalhöyük comme mise en scène}

Une douzaine d'années après avoir défini la «prémisse de Pompéi », Ascher en vint assez naturellement à s'intéresser à l'engouement de ses contemporains pour les capsules temporelles (Ascher 1974). Selon lui, cellesci dénoteraient en effet une double méprise: d'un côté, les sites archéologiques les mieux préservés seraient supposés agir comme des capsules temporelles; de l'autre, les capsules temporelles seraient, pour les archéologues du futur, porteuses d'un témoignage d'autant plus représentatif qu'elles sont intentionnelles. Or le caractère inopiné du site archéologique «idéal» s'oppose évidemment au caractère planifié d'une capsule. Mais derrière cette contradiction se cache une similarité plus profonde. L'inévitable déception qui accompagne l'ouverture d'une capsule est finalement assez analogue aux frustrations croissantes qu'éprouvent les archéologues à mesure qu'ils réalisent qu'un site bien préservé n'est pas plus transparent pour autant. Dans les deux cas, le même décalage persiste entre les attentes et l'expérience. Les capsules temporelles seraient ainsi le pendant symétriquement inverse de la «prémisse de Pompéi »: le fantasme d'un arrêt sur image à un point $\mathrm{T}$.

En conclusion de l'article, Ascher est donc amené à douter de la fonction archéologique proclamée des capsules temporelles. Mais il est possible de suivre ce même raisonnement dans l'autre sens. Certes la capsule temporelle n'est le type idéal du site archéologique qu'en vertu d'une illusion, mais elle constitue, en fin de compte, une modalité de production de traces parmi d'autres. Si l'on met de côté le fait, ici accessoire, que les capsules temporelles contemporaines sont généralement (mais pas toujours) programmées pour être ouvertes à une date précise ${ }^{8}$, on pourrait ainsi qualifier tous contextes dont le statut interprétatif est moins lié à une condition rétrospective de vestiges qu'à la part d'intention prospective dans leurs conditions initiales d'existence et de préservation. Cette redéfinition archéologique de la capsule temporelle permettrait, autrement dit, de distinguer les restes qui sont le produit d'une intentionnalité taphonomique. Et si une telle catégorie de vestiges ne remplira certainement pas les objectifs chimériques que l'on se plaît à attribuer aux capsules temporelles de la modernité, la trace ainsi produite possédera sans conteste cette étrangeté propre aux messages que l'on devine cryptés derrière une apparente familiarité.

Appliquée à des sites archéologiques, la notion de capsule temporelle évoque généralement des lieux qui, comme une ville ensevelie sous des nuées ardentes ou une grotte scellée par un éboulement, auraient été préservés des épreuves du temps. Dans ce sens, Çatalhöyük a bel et bien sa place aux côtés de Chauvet ou de Pompéi. Mais lorsqu'elle est ainsi rétrospectivement projetée sur la préhistoire, la notion se charge d'un bagage supplémentaire, 


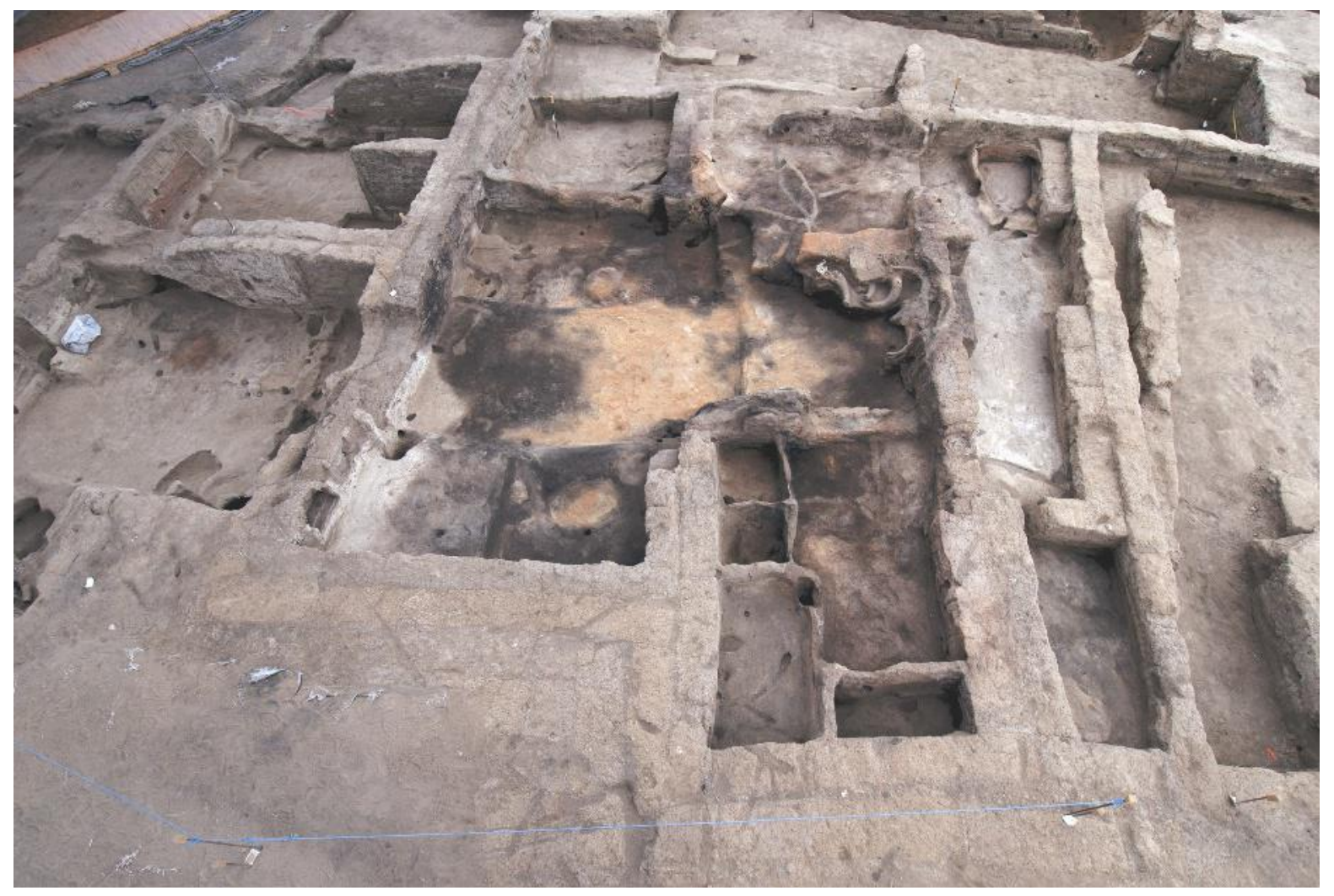

fig. 7

Le bâtiment 52 avant la reprise de la fouille.

À droite, la superposition de murs correspond

à un épisode de reconstruction, probablement

en réponse à une instabilité structurelle.

Tout en haut, on distingue les restes très

érodés du four circulaire en tannour. Un peu

plus bas, le crâne d'aurochs et la banquette

ornée de cornes. En bas à droite, les silos

permettaient de préserver céréales,

légumineuses ou oléagineuses. À gauche,

les plateformes accueillaient les inhumations.

L'emplacement de la sépulture multiple

se dessine ici clairement d'une couleur

différente car non recouverte d'enduit, 2008.

Photo () Jason Quinlan. 
caractéristique de l'obstacle épistémologique généré par l'illusion référentielle: les capsules temporelles archéologiques apparaissent toutes accidentelles, comme le reflet d'un rapport passif à l'événement (Jarvis 2002: 22-24). Or, contrairement à Chauvet ou Pompéi, Çatalhöyük n'est une capsule temporelle qu'en vertu de pratiques culturelles. La métaphore adéquate serait plus à chercher du côté de l'inhumation funéraire que de la catastrophe sans lendemain. Çatalhöyük est, à cet égard, bien plus proche de ce que I'on qualifie au sens strict de capsule temporelle pour l'époque contemporaine. Deux aspects essentiels de cette notion doivent ici être réintégrés à l'interprétation: la dimension réflexive, qui fait de chaque capsule temporelle l'objet d'une sélection de ce qui doit rester ou de ce qu'il faut léguer; et la dimension performative, qui voit les acteurs produire une trace pour le futur, indépendamment de sa destination exacte, à la faveur d'un geste significatif en lui-même. En replaçant l'intention au cœur de l'action, la notion de capsule temporelle pose à nouveaux frais - indépendamment de l'horizon interprétatif de l'occupation - la question de la représentativité. Et en exigeant d'aborder ces vestiges pour moins transparents qu'ils n'y paraissent, comme s'ils étaient en quelque sorte «codés» par les choix de leurs auteurs, elle peut servir de clef pour en dé-familiariser l'approche et les réinvestir ainsi d'une «étrangeté » plus heuristique d'un point de vue anthropologique.

II est difficile de déterminer les raisons qui poussaient les habitants de Çatalhöyük à détruire régulièrement le haut de leurs «maisons » pour reconstruire un nouveau bâtiment parfois exactement superposé à l'ancien. Le rythme même de ces pratiques est difficile à estimer. La quantité d'épisodes de réfection (plusieurs centaines sur certains murs, Matthews 2005) et la datation absolue (malheureusement trop imprécise pour cette période, Cessford 2005) dénotent des durées d'occupation longue, plus d'un demi-siècle en moyenne, sans qu'il soit toutefois possible de distinguer une véritable régularité à l'échelle du site. Mellaart privilégiait la thèse de l'obsolescence pour expliquer ces démolitions (1967: 50). De tels problèmes sont en effet courants sur un tell composé de couches de débris superposés et donc propices à des affaissements irréguliers. Mais les habitants de Çatalhöyük semblent avoir parfaitement intégré cette variable à leur pratique de construction. Au-delà du caractère plastique d'une architecture de terre en continuelle transformation, les structures peuvent être l'objet d'interventions lourdes qui nécessitaient parfois de démonter une partie de la charpente. Un mur extérieur du bâtiment 52 a ainsi été entièrement reconstruit tandis qu'une pièce était intégralement remplie de remblais au cours de l'occupation, probablement en réponse à des défaillances structurales (fig. 7). Autrement dit, la reconstruction partielle du bâtiment peut se substituer, s'il le faut, à sa démolition: aucune raison de douter, donc, des capacités des habitants de Çatalhöyük à prolonger l'usage des bâtiments sur de longues périodes et à déterminer le moment exact de leur destruction.

II reste toutefois malaisé d'aller plus loin dans la définition des intentions. Il est certes tentant d'associer, par exemple, la condamnation du bâtiment 52 au dépôt funéraire qui l'accompagne, ou d'y voir une explication aux circonstances particulières qui entourent sa destruction par un incendie volontaire, mais cette relation n'a rien d'univoque. Seuls deux des individus de la sépulture multiple, l'adulte et le nourrisson, étaient dans un état compatible avec une mort récente au moment de l'inhumation. La simultanéité des décès était-elle fortuite ou provoquée? En quoi est-elle liée à la décision 


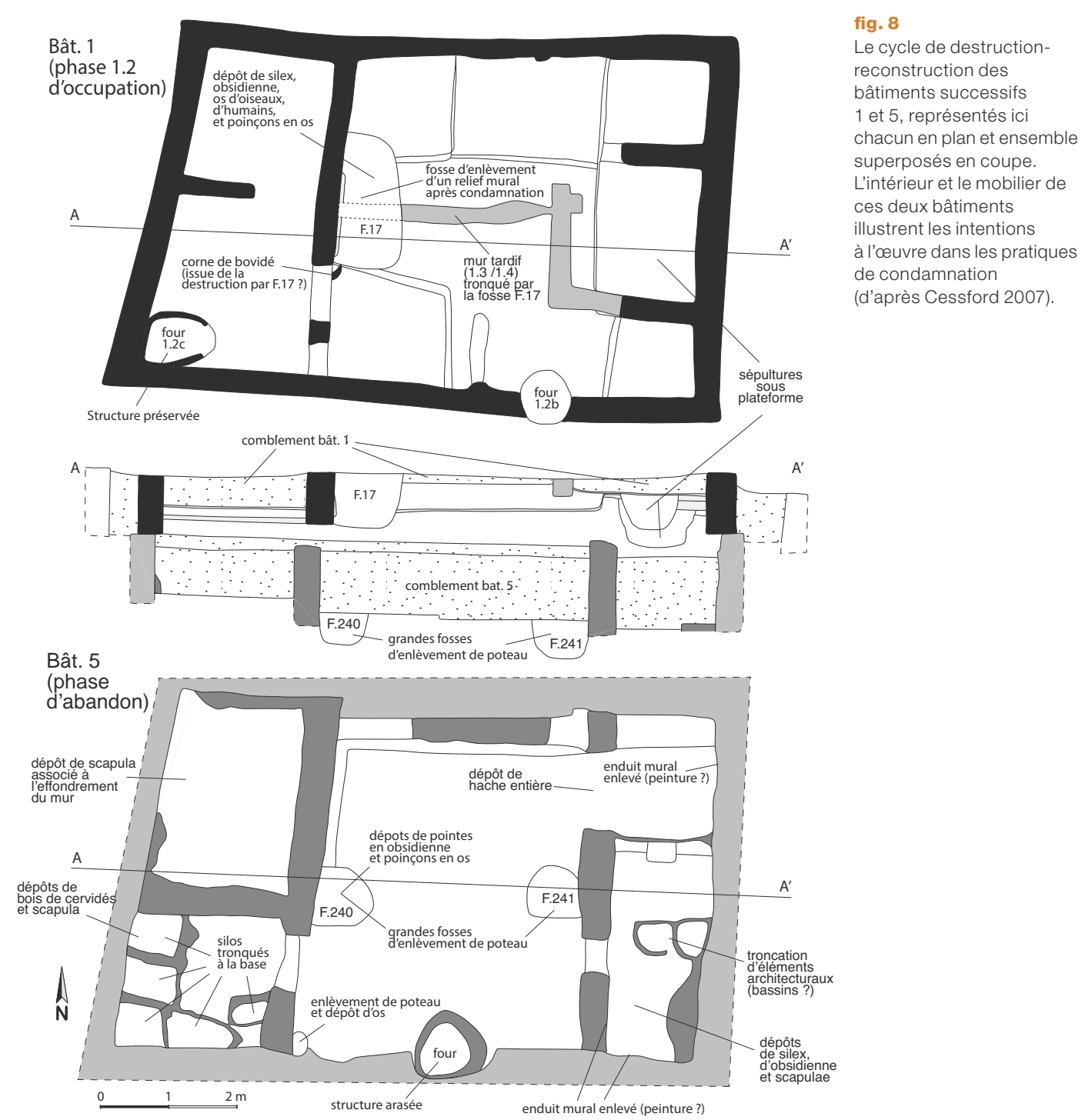

de détruire le bâtiment? Et s'il y a causalité, dans quel sens fonctionne-t-elle ${ }^{9}$ ? Prise dans sa totalité, la sépulture complexifie encore les termes de la question. Les squelettes incomplets des autres enfants qui y sont regroupés suggèrent en effet que les corps en décomposition ont préalablement transité, soit par une première sépulture, soit par un autre lieu de «stockage» provisoire. En prévision de leur réunion dans la ruine du bâtiment 52 ? La succession des actions funéraires qu'il a été possible de retracer sur la plateforme voisine pourrait étayer l'hypothèse d'une forme d'anticipation. Le crâne d'un individu enterré au moment de la construction y a été prélevé lorsqu'une deuxième inhumation recoupa l'ancienne pendant l'occupation du bâtiment. L'objet dut être soigneusement conservé, son origine et sa destination considérées comme particulièrement importantes, puisqu'il fut plus tard réintroduit dans une troisième sépulture réalisée au même endroit, peu de temps avant l'incendie (Haddow et al. 2016).
9. La possibilité d'une mise à mort de l'adulte ou de l'enfant à l'occasion de la condamnation du bâtiment peut paraître audacieuse. Elle tranche notamment avec l'image idéalisée d'une société égalitaire et pacifique régulièrement projetée sur Çatalhöyük. L'hypothèse mérite néanmoins d'être considérée au regard des pratiques articulant destruction architecturale et exécution ritualisée à l'époque précédente du Néolithique précéramique (Hadad 2019). 
10. II s'agit, plus exactement, et pour tous les cas suivants, de chevilles osseuses, c'est-à-dire de la partie du crâne qui se prolonge à l'intérieur de la corne, cette dernière ne se conservant pas.
L'acte paraît également planifié dans la mesure où il concernait certainement une bonne partie de la communauté. Bien qu'agglutinés, les bâtiments de Çatalhöyük ne possèdent pas de murs mitoyens. Chaque cycle de destruction-construction peut donc suivre un rythme propre. Mais cet argument, souvent avancé en faveur de l'hypothèse de «maisonnées» indépendantes, n'enlève rien à la dimension collective de ces épisodes. La solidarité structurale de bâtiments qui reposaient souvent les uns sur les autres impliquait nécessairement l'ensemble du voisinage, tandis que l'échelle des travaux de démontage, de remblaiement et de reconstruction mobilisait probablement au-delà des seuls résidents. L'acte en lui-même ne visait d'ailleurs pas une seule efficacité techno-économique. Si le recyclage des matériaux était une pratique courante, il paraît toujours subordonné à d'autres considérations. La préciosité du bois de construction, par exemple, explique aisément que l'on retire les poteaux avant le comblement, mais également que la charpente serve malgré tout de combustible à l'occasion d'un incendie volontaire. Similairement, l'arasement des murs possède certes une fonction tectonique en procurant des fondations solides et des briques réutilisables pour un nouveau bâtiment, mais des murs conservés sur une hauteur moindre auraient fourni davantage d'adobe et économisé une partie du travail de remplissage sans constituer pour autant de plus mauvaises fondations.

D'un point de vue taphonomique, donc, les pratiques de condamnation se caractérisent par un régime culturel de la destruction soucieux de ce qui reste (Hodder 2005: 134-135; Farid 2007: 52-53). Les assemblages associés aux derniers niveaux d'occupation sont, pour une large partie du moins, directement fonction de la mise en œuvre de ces épisodes, soit parce que leur localisation (au fond d'un trou de poteau arraché, au niveau d'un seuil ou par-dessus un foyer ou un silo), leur agencement (parfois soigné) et leur nature (restes humains et animaux, artefacts entiers) suggèrent des dépôts volontaires (fig. 8), soit parce qu'ils correspondent aux reliefs de repas collectifs abandonnés sur place au moment de la destruction (Russell et al. 2014). L'attention, toutefois, se focalise sur les éléments architecturaux. L'enlèvement préférentiel des parties considérées comme étant les plus symboliquement investies a été depuis longtemps souligné (Mellaart 1967: 82, 102-103). Si les peintures murales étaient facilement recouvertes par un nouvel enduit (comme elles l'étaient d'ailleurs tout au long de l'occupation), les murs font parfois l'objet d'interventions plus destructives. Des fragments de cornes ${ }^{10}$ partiellement enduites sur les derniers sols d'occupation suggèrent le retrait des «trophées» intégrés à l'architecture (Twiss et Russell 2009: 26-27). Mais le filtre culturel derrière ces pratiques n'est nulle part plus saillant que dans les célèbres figures anthropomorphes façonnées en relief sur les murs. Toutes ont été mutilées au moment du comblement. La tête, les pieds et les mains sont la cible systématique d'un geste qui épargne généralement le reste du corps (fig. 9). Un caractère essentiel qu'ignorent souvent les tentatives d'y voir, sur la base de reconstructions pourtant douteuses, la représentation d'une Déesse-Mère, d'un léopard ou, plus récemment, d'un ours. Mais sa prise en compte peut également masquer un déficit plus profond de raisonnement taphonomique, comme lorsqu'Alain Testart fait des cavités laissées par les mutilations des emplacements destinés à accrocher la tête et les pattes d'un léopard chassé et attribue ainsi à l'occupation du bâtiment une caractéristique directement imputable à sa destruction (Testart 2010: 55). 


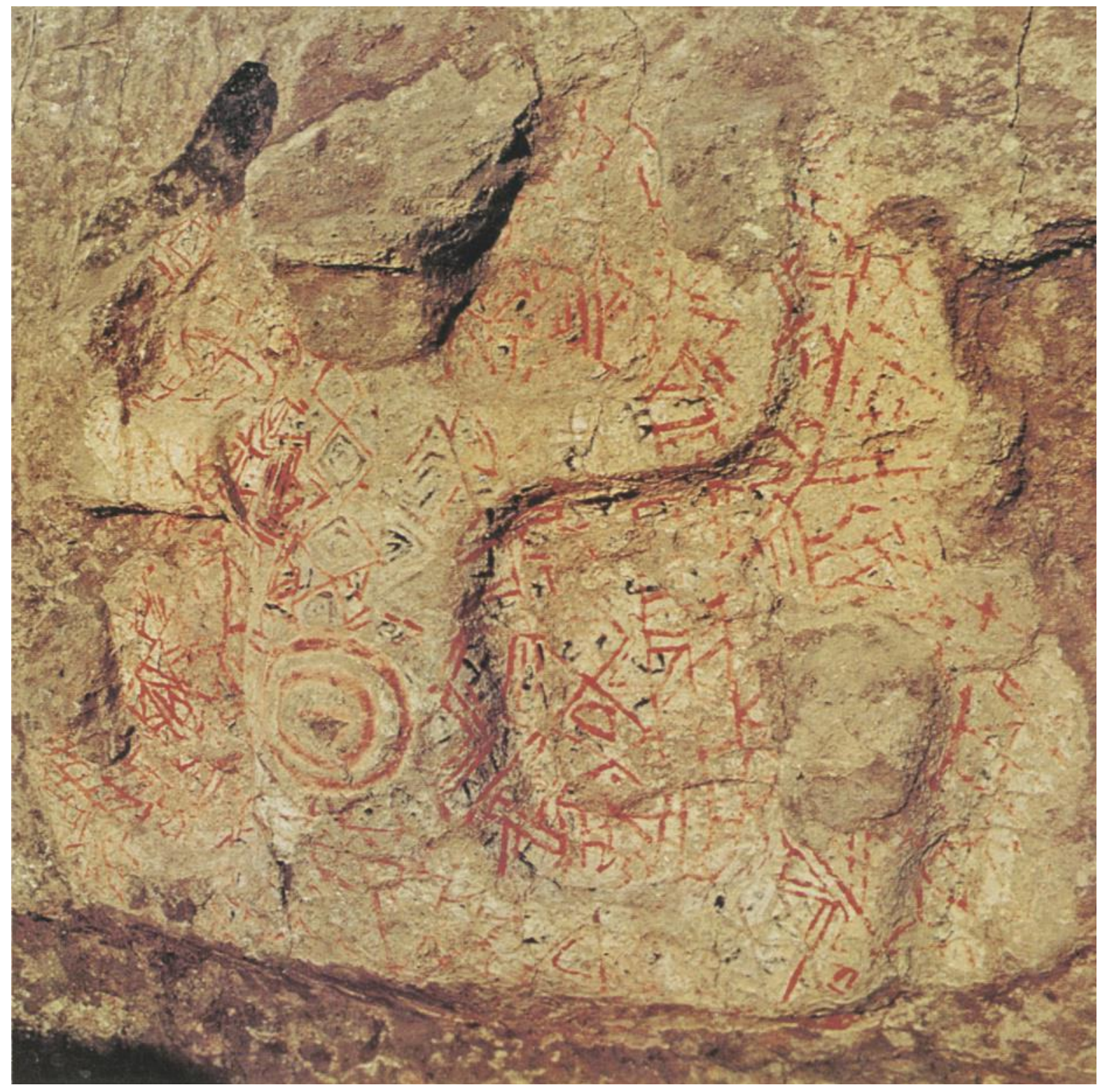

fig. 9

L'une des célèbres figures éployées,

d'abord qualifiée de Déesse-Mère par

Mellaart, et aujourd'hui comparée à

une parturiente, à un fauve ou à un ours.

Cet exemplaire, l'un des mieux conservés,

ne fait pas exception parmi la dizaine

d'autres découverts par Mellaart: tous

ont vu au minimum leur tête et l'extrémité

de leurs membres mutilées avant

le comblement du bâtiment

(Mellaart 1967, pl. VII). Droits réservés. 


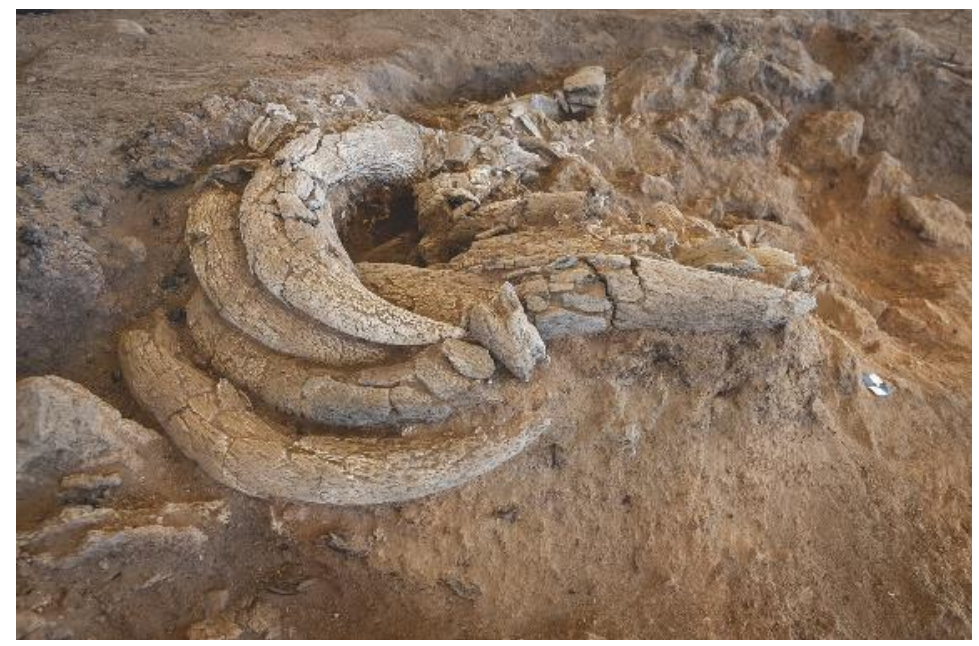

a

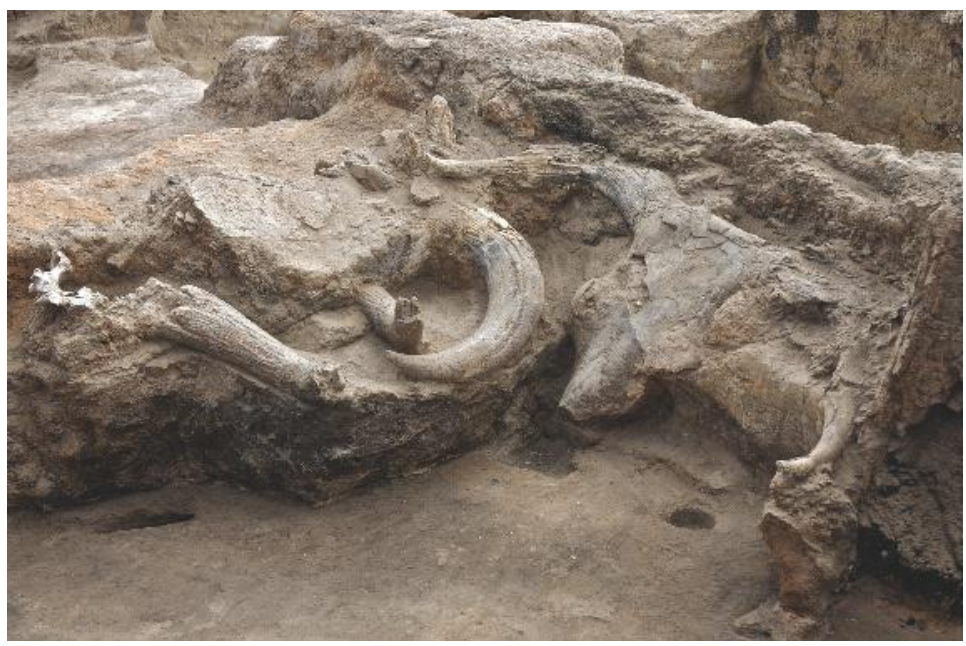

fig $10 a, b$ et c

Séquence d'accrétion d'une même installation dans le bâtiment 52: a - au cours de l'occupation du bâtiment, plusieurs massacres d'ovins et de caprinés sauvages sont installés dans un réceptacle en terre; $b$ - une banquette dans laquelle sont fichées trois grandes cornes de bovin vient ensuite recouvrir, sans la détruire, l'installation précédente (le crâne d'aurochs était peut-être déjà présent) ; c-au moment de la destruction du bâtiment, treize cornes d'aurochs, peut-être collectées sur d'autres installations du bâtiment, sont soigneusement déposées en faisceau sur l'ensemble, préalablement protégé de l'incendie par un comblement particulier. Photos (c) Jason Quinlan.

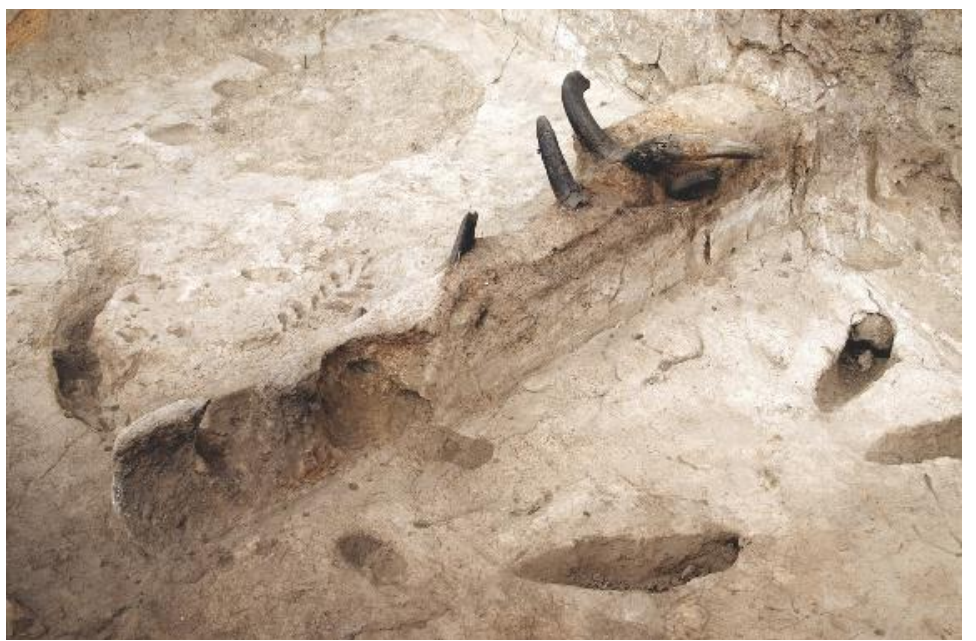


Inversement, d'autres éléments semblent avoir été intentionnellement préservés, voire activement «renforcés». Ainsi, les fours et les silos, souvent soigneusement arasés pour y récupérer des argiles aux propriétés plastiques particulières, sont parfois consciencieusement remplis afin de ne pas être écrasés par le poids des remblais (Hodder 2006: 132). Dans le bâtiment 52, le crâne d'aurochs a très peu souffert de l'incendie pourtant particulièrement intense dans cette partie du bâtiment (Harrison et al. 2013; Farid 2014). II a sans doute été protégé par un remblaiement partiel avant la mise à feu. Un peu au-dessus, dans le comblement, treize cornes d'aurochs calcinées ont été soigneusement rassemblées en un faisceau bien ordonné. Il est impossible de déterminer si ces objets, peut-être issus du démantèlement d'autres installations, ont été déposés avant ou après l'incendie, mais leur localisation est sans conteste en lien avec la préservation du crâne sous-jacent. Juste à côté de ce dernier, trois grandes cornes d'aurochs insérées dans une petite banquette ont été également abandonnées in situ lors du comblement. II s'agit en fait du second état d'une installation auparavant plus petite accueillant des massacres d'ovins et de caprins sauvages. Lors d'un épisode de réfection, la «banquette » aux aurochs est donc venue recouvrir et intégrer les cornes précédentes sans les démanteler (fig 10a, b et c). La destruction prolonge donc ici une séquence d'accrétion observée au cours de l'occupation, comme s'il s'agissait d'une réfection supplémentaire, et participe à un «renforcement» non seulement de structure mais aussi de substance. Ailleurs, les modalités de destruction/préservation sont dictées par la continuation de l'occupation dans un nouveau bâtiment. Ainsi qu'il est parfois d'usage pour collecter des restes humains inhumés sous des maisons, des fosses peuvent être creusées a posteriori pour détruire ou récupérer une installation enterrée (Hodder 2006: 144-145).

Derrière l'image figée se profile ainsi peu à peu «l'état de changement perpétuel qui caractérise chaque communauté vivante» sur l'omission duquel la «prémisse de Pompéi » s'établit. À Çatalhöyük, cette temporalité négligée a la particularité d'être à la fois répétitive et cumulative. Lorsqu'un bâtiment est édifié immédiatement au-dessus d'un ancien, il hérite généralement de sa configuration spatiale interne. Cette continuité peut se perpétuer sur plusieurs cycles au point que ceux-ci apparaissent parfois davantage comme la reproduction d'une même entité que comme autant de ruptures dans la biographie respective des structures. Au cours de ces séquences, certaines de ces «colonnes» de bâtiments semblent capitaliser sur cette accumulation en concentrant une quantité croissante d'attributs mémoriels, tels que les restes humains ou les «trophées » animaux. Ce phénomène a pu être interprété comme un processus de différenciation par la production et la localisation d'une « histoire » (Hodder et Pels 2010) ou comme l'affirmation de la «maison» en tant que principe pérenne d'organisation sociale (Bloch 2010). Mais, plus qu'une accumulation progressive dont les bénéfices ne seraient appréciés que rétrospectivement, la somme des actions mérite également d'être examinée à l'échelle des gestes. Webb Keane a ainsi suggéré d'y voir «un effort conscient de contrôle » exercé sur la force évocatrice du bâtiment et de son contenu par la maîtrise «des transitions entre visible et invisible, présence et absence» (Keane 2010: 208-211).

Cette dernière approche a ceci d'intéressant qu'elle met les restes de la destruction architecturale en perspective avec la capacité des acteurs à anticiper le rôle des traces ainsi produites. Produire activement du passé, 
pour le dire autrement, c'est aussi agir au futur. Les habitants de Çatalhöyük sont ainsi supposés manipuler un invisible qui demeure signifiant parce qu'ils le savent présent, à la fois préservé et dissimulé sous l'établissement. Ensevelir s'apparenterait alors à l'exercice d'un pouvoir-soustraire, à une manière d'attirer l'attention par la dramatisation d'une absence, dont l'expérience est entretenue par les pratiques régulières de réfection architecturale et d'inhumation funéraire, et dont l'empreinte s'actualise dans la physionomie à la fois constante et changeante du bâti. La condamnation des bâtiments apparaît comme cruciale car elle articule cette réalité tangible à un phénomène de plus longue durée. La croissance du tell est en effet presque entièrement tributaire de ces épisodes ritualisés d'inhumation architecturale. Leur répétition dans le temps propre des dynamiques de destructions/préservation/reconstructions, comme dans l'espace «agglutinant » du site produit, à l'échelle de la plaine environnante, une colline anthropique, un artefact culturel unique et démesuré qui n'avait sans doute rien de fortuit aux yeux des habitants. Si l'on prend au sérieux leurs actions, y compris dans ce qu'elles ont de prospectif, le tell apparaît moins comme le sous-produit passif de l'occupation que comme la matérialisation consciente, si ce n'est souhaitée et anticipée, du renouvellement continuel de l'espace vécu.

Comment - donc - conserver ce qui n'a pas été construit pour durer? La question que se posent aujourd'hui les archéologues n'est pas totalement hors de propos pour saisir le rapport que les habitants de Çatalhöyük entretenaient avec le site. Eux aussi s'attachaient, à leur manière, à prolonger dans le temps l'intégrité des bâtiments abandonnés. Mais ils appliquaient au problème une solution difficile à concevoir pour qui tenterait seulement de déceler, dans les scènes exhumées, la manifestation d'un présent figé dans le passé. La notion de mise en scène vient assez naturellement à l'esprit pour caractériser l'artifice que constitue à cet égard un contexte rituellement clos et ordonné (Le Brun 2003). Mais elle décrit également bien la méprise qui s'installe alors entre les attentes de l'archéologue et l'intention des Néolithiques. Car elle circonscrit ce qui, dans le geste des seconds, expose le premier à l'illusion référentielle lorsqu'il plaque sur les vestiges une temporalité qui n'est pas la leur. Reconnaître l'importance, pour les habitants de Çatalhöyük, d'un passé présentifié à même le tell est un premier pas, mais il n'est pas suffisant. Le regard rétrospectif a en effet tendance à restreindre la capacité des Néolithiques à anticiper les résultats de leurs propres actions. Or dans un monde marqué par la re-production active et régulière de l'espace vécu, où une sélection minutieuse s'opère sur ce qui peut être ainsi inhumé, et où les objets préservés le sont souvent dans un jeu de miroir avec les constructions à venir, la question du futur ne peut être ignorée, ne serait-ce que pour restituer un mouvement dont les vestiges sont l'expression directe. En rompant avec la «prémisse de Pompéi » et la reconstruction d'instantanés archéologiques, la notion de capsule temporelle comme modalité de production de vestiges permet de rendre compte de la question de l'action et de son telos. L'intentionnalité taphonomique responsable de la bonne préservation des «maisons» néolithiques ne génère pas tant du passé, au sens d'une réalité référant à un temps révolu, qu'un présent idéalisé en fonction d'un «horizon d'attente » dont le tell constituait, déjà, une manifestation tangible (Koselleck 2016). Le propre de Çatalhöyük en la matière, c'est que ces actions y ont généralisé 
ce principe de la capsule temporelle à l'échelle du site. À force de répétition et d'accumulation, elles le produisent même presque entièrement. Et si l'on ne saurait évidemment calquer leur destination sur celle de leurs équivalents contemporains, ce qui laisse en suspens la question délicate des représentations du temps et des conceptions de l'avenir, leur double dimension réflexive et performative permet néanmoins de penser des productions prospectives qui, comme toutes les capsules temporelles, s'adressaient avant tout à leurs auteurs.
Université Paris Nanterre, département d'anthropologie, UMR 7055 Préhistoire et Technologie remi.hadad@mae.u-paris10.fr
Remerciements :

Je remercie lan Hodder de m'avoir accordé sa confiance, Burcu Tung et l'ensemble des personnes qui ont pris part à la fouille du bâtiment 52 (tout particulièrement Maciej Chyleński, Christoffer Hagberg et Paul Pettersson). Merci également à Emmanuel Grimaud pour ses commentaires sur une précédente version de ce texte et à Frédéric Keck pour ses encouragements tout au long de ce travail. 
Bibliographie

\section{Ascher, Robert}

1961 «Analogy in Archaeological Interpretation ", Southwestern Journal of Anthropology 17 (4): 317-325.

1968 "Time's Arrow and the Archaeology of a Contemporary Community ", in Kwang-Chih Chang (dir.), Settlement Archaeology. Palo Alto, National Press Books: 47-79.

1974 "How to build a Time Capsule", Popular Culture 8 : 241-253.

\section{Bachelard, Gaston}

1938 La Formation de l'esprit scientifique. Contribution à une psychanalyse de la connaissance. Paris, Vrin.

\section{Baird, Douglas}

2005 "The History of Settlement and Social Landscapes in the Early Holocene in the Çatalhöyük Area ", in lan Hodder (éd.), Çatalhöyük Perspectives. Cambridge, McDonald Institute for Archaeological Research: 55-74.

\section{Barthes, Roland}

1968 "L'effet de réel »,

Communications 11 : 84-89.

Bayliss, Alex, Brock, Fiona, Farid, Shahina, Hodder, lan, Southon, John et Taylor, R.E.

2015 « Getting to the Bottom of it all: A Bayesian Approach to Dating the Start of Çatalhöyük», Journal of World Prehistory 28 (1): 1-26.

\section{Binford, Lewis}

1981 "Behavioral Archaeology and the "Pompeii Premise" ", Journal of Anthropological Research 37 (3) : 195-208

\section{Bloch, Maurice}

2010 «Is there Religion at Çatalhöyük... or are there just Houses? ", in lan Hodder (dir.) Religion in the Emergence of Civilization. Çatalhöyük as a Case Study. Cambridge, Cambridge University Press: 146-162.

\section{Bogaard, Amy et al.}

2009 «Private Pantries and Celebrated Surplus: Storing and Sharing Food at Neolithic Çatalhöyük, Central Anatolia ", Antiquity 83 (321): 649-668.

Une illusion du vraisemblable. Mise en scène taphonomique et prospective néolithique à Çatalhöyük. Par Rémi Hadad

\section{Bordes, François}

1975 «Sur la notion de sol d'habitat en préhistoire paléolithique *, Bulletin de la Société préhistorique francaise 72 : 139-144.

\section{Boz, Başak et Hager, Lori}

2013 «Intramural Burial Practices at Catalhöyük, Central Anatolia, Turkey ", in lan Hodder (éd.), Humans and Landscapes of Çatalhöyük. Reports from the 2000-2008 Seasons. Los Angeles, Cotsen Institute of Archaeology: 413-40.

\section{Buchli, Victor et Lucas,} Gavin

2001 "The Absent Present", in Victor Buchli et Gavin Lucas (éd.), Archaeologies of the Contemporary Past. Londres/New York, Routledge: 3-18

\section{Cauvin, Jacques}

1997 Naissance des divinités. Naissance de l'agriculture. La Révolution des symboles au Néolithique. Paris, Flammarion.

\section{Cessford, Craig}

2003 « Microartifactual Floor Patterning: The Case at Çatalhöyük », Assemblage 7.

2005 «Absolute Dating at Çatalhöyük ", in lan Hodder (éd.), Inhabiting Çatalhöyük. Reports from the 1995-99 Seasons. Cambridge, McDonald Institute for Archaeological Research : 65-99.

2007 "Neolithic Excavation in the North Area ", in lan Hodder (éd.), Excavating Catalhöyük. South, North and Kopal area Reports from the 1995-1999 Seasons. Cambridge, McDonald Institute for Archaeological Research : 345-532.

\section{Cessford, Craig et Near, Julie}

2005 «Fire, Burning and Pyrotechnology at Çatalhöyük», in lan Hodder (éd.), Çatalhöyük Perspectives. Reports from the 1995-1999 Seasons. Cambridge, McDonald Institute for

Archaeological Research: 171-182.

\section{Düring, Bleda}

2006 Constructing Communities. Clustered Neighbourhood Settlements of the Central Anatolia Neolithic, ca. 8500-5500 Cal. BC. Leiden, Nederlands Instituut Voor Het Nabije Oosten.
2007 «Reconsidering the Çatalhöyük Community: From Households to Settlement Systems ", Journal of Mediterranean Archaeology 20 (2) : 155-182.

\section{Farid, Shahina}

2007 «Introduction to South Area Excavations", in lan Hodder (éd.), Excavating Çatalhöyük. South, North and Kopal Area Reports from the 1995-1999 Seasons : 41-58.

2014 "Buildings 52/51", in lan Hodder (éd.), Çatalhöyük Excavations. The 2000-2008 Seasons. Los Angeles, Cotsen Institute of Archaeology: 357-398.

\section{Freud, Sigmund}

1985 L'Inquiétante étrangeté et autres essais, trad. de l'allemand par F. Cambon. Paris, Gallimard.

2013 Le Délire et les rêves dans "Gradiva » de W. Jensen, trad. de l'allemand par D. Tassel. Paris, Points.

\section{Girard, René}

2015 "Animal Scapegoating at Çatalhöyük ", in Pierpaolo Antonello et Paul Gifford (éd.). How We Became Human. Mimetic Theory and the Science of Evolutionary Origins. East Lansing, Michigan State University Press : 217-232.

\section{Hadad, Rémi}

2019 «Ruin Dynamics: Architectural Destruction and the Production of Sedentary Space at the Dawn of the Neolithic Revolution ", Journal of Social Archaeology 19 (1).

\section{Haddow, Scot} Sadvari, Joshua, Knüsel, Christopher et Hadad, Rémi

2016 «A Tale of Two Platforms: Commingled Remains and the Life-Course of Houses at Neolithic Çatalhöyük », in Anna Osterholtz (éd.), Theoretical Approaches to Analysis and Interpretation of Commingled Human Remains, New York, Springer : 5-29.

\section{Harrison, Karl, Martin, Victoria et Webster, Brooke}

2013 «Structural Fires at Çatalhöyük», in lan Hodder (éd.), Substantive Technologies at

Catalhöyük. Reports from the 2000-2008 Seasons. Los Angeles, Cotsen Institute of Archaeology Press: 137-146.

\section{Hodder, Ian}

1990 The Domestication of Europe. Structure and Contingency in Neolithic Societies. Oxford, Basil Blackwell.

2005 "The Spatio-temporal Organization of the Early "Town" at Çatalhöyük", in Douglass Bailey, Alasdair Whittle et Vicki Cummings (éd.), (Un)settling the Neolithic. Oxford, Oxbow Books: 126-139.

2006 Çatalhöyük the Leopard's Tale. Revealing the Mysteries of Turkey's Ancient "Town ». Londres, Thames \& Hudson.

2014 "Çatalhöyük: The Leopard Changes its Spots. A Summary of Recent Work", Anatolian Studies 64 $1-22$

\section{Hodder, Ian et Pels, Peter}

2010 "History Houses:

A New Interpretation of Architectura Elaboration at Çatalhöyük», in lan Hodder (éd.), Religion in the Emergence of Civilization. Catalhöyük as a Case Study. Cambridge, Cambridge University Press: 163-186.

\section{Jarvis, William}

2002 Time Capsules. A Cultural History. Jefferson, McFarland \& Co.

\section{Keane, Webb}

2010 "Marked, Absent, Habitual. Approaches to Neolithic religion at Çatalhöyük», in lan Hodder (éd.), Religion in the Emergence of Civilization. Çatalhöyük as a Case Study. Cambridge, Cambridge University Press: 187-219.

\section{Koselleck, Reinhart}

2016 Le Futur passé. Contributions à la sémantique des temps historiques, trad. de l'allemand par J. et M.-C. Hoock. Paris, Éditions de I'EHESS. 


\section{Le Brun, Alain}

2003 «Idéologie et symboles

à Khirokitia : la "fermeture"

d'un bâtiment et sa mise en

scène ", in Jean Guilaine et Alain

Le Brun (éd.), Le Néolithique de

Chypre. Athènes, École française

d'Athènes: 341-349.

\section{Lewis-Williams, David et Pearce, David}

2005 Inside the Neolithic Mind. Consciousness, Cosmos and the Realm of the Gods. Londres,

Thames \& Hudson.

\section{Matthews, Wendy}

2005 «Micromorphological and Microstratigraphic Traces of Uses and Concepts of Space", in Ian Hodder (éd.), Inhabiting Chatalhöyük. Reports from the 1995-99 Seasons. Cambridge, McDonald Institute for Archaeological Research: 355-398.

\section{Mellaart, James}

1964 « Excavations at Catal Hüyük, 1963: Third Preliminary Report ", Anatolian Studies 14 39-119.

1967 Çatal Hüyük. A Neolithic Town in Anatolia. Londres, Thames \& Hudson.

1975 The Neolithic of the Near East. Londres, Thames \& Hudson.

\section{Russell, Nerissa et al.}

2014 "Bringing down the House: House closing Deposits at Çatalhöyük», in lan Hodder (éd.), Integrating Catalhöyük. Themes from the 2000-2008 Seasons. Los Angeles, Cotsen Institute of Archaeology: 109-121.

\section{Schiffer, Michael}

1985 « Is There a "Pompeii Premise" in Archaeology?", Journal of Anthropological Research 41: 18-41.

\section{Stevanović, Mirjana}

1997 "The Age of Clay: The Social Dynamics of House Destruction ", Journal of Anthropological Archaeology 16 (4) : 334-395

\section{Testart, Alain}

2010 La Déesse et le grain.

Trois essais sur les religions néolithiques. Paris, Errance.

\section{Tringham, Ruth}

2000 "The Continuous House: A View from the Deep Past ", in Rosemary Joyce et Susan Gillespie (éd.), Beyond Kinship. Philadelphie, University of Pennsylvania Press: 115-134.

2013 «Destruction of Places by Fire: Domicide or Domithanasia ", in Jan Driessen (éd.), Destruction. Louvain, Presses universitaires de Louvain : 89-108.

\section{Twiss, Katheryn et al.}

2008 «Arson or Accident? The Burning of a Neolithic House at Çatalhöyük, Turkey ", Journal of Field Archaeology 33 (1): 41-57.

2009 «Plants and Animals Together: Interpreting Organic Remains from Building 52

at Çatalhöyük", Current Anthropology 50 (6) : 885-895.

\section{Twiss, Katheryn et Russell, Nerissa}

2009 «Taking the Bull by the Horns: Ideology, Masculanity, and Cattle Horns at Çatalhöyük ", Paléorient 35 (2) : 19-32.

\section{Verhoeven, Marc}

$\mathbf{2 0 0 0}$ «Death, Fire and Abandonment: Ritual Practice at late Neolithic Tell Sabi Abyad, Syria ", Archaeological Dialogues 7: 46-65.

\section{Vidler, Anthony}

1992 The Architectural Uncanny. Essays in the Modern Unhomely. Cambridge, The MIT Press.

\section{Weismantel, Mary}

2014 "The Hau of the House", in lan Hodder (éd.), Religion at Work in a Neolithic Society. Vital Matters. Cambridge, Cambridge University Press: 259-279.

\section{Wright, Katherine}

2014 «Domestication and Inequality? Households, Corporate Groups and Food Processing Tools at Neolithic Çatalhöyük", Journal of Anthropological Archaeology 33: 1-33. page 112 et ci-contre Jason Quinlan, La «banquette» aux aurochs, site de Çatalhöyük, Turquie, 2008 (détail) Photo () Jason Quinlan.

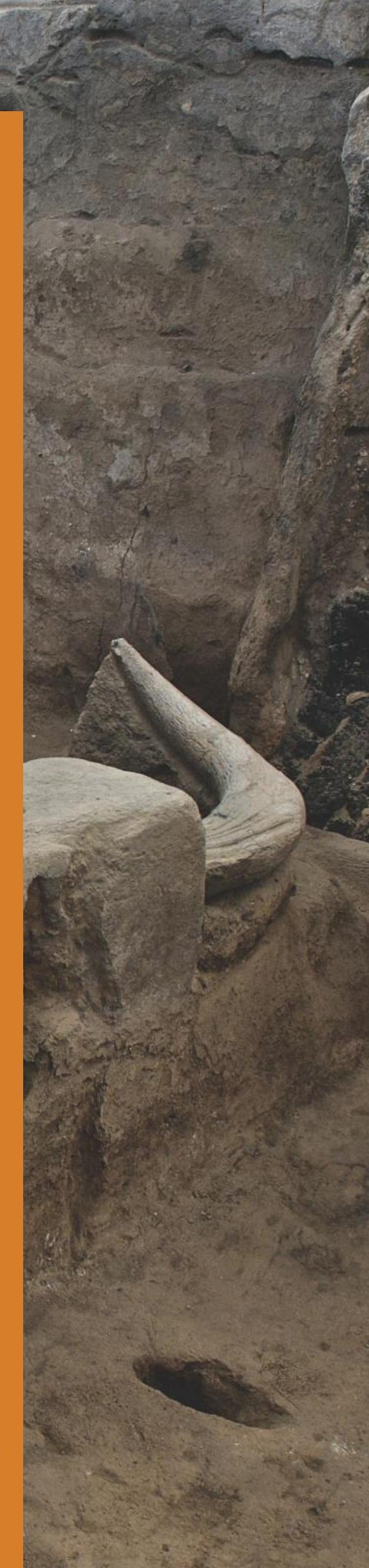

